\title{
WMAP constraints on SUGRA models with non-universal gaugino masses and prospects for direct detection.
}

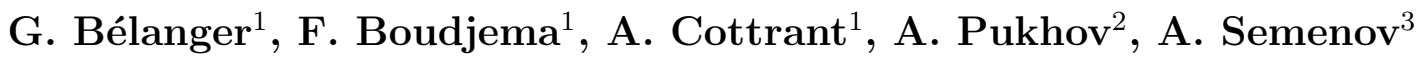 \\ 1. Laboratoire de Physique Théorique $\mathrm{LAPTH}^{1}$ \\ Chemin de Bellevue, B.P. 110, F-74941 Annecy-le-Vieux, Cedex, France. \\ 2. Skobeltsyn Institute of Nuclear Physics, Moscow State University \\ Moscow 119992, Russia \\ 3. Joint Institute for Nuclear Research (JINR) \\ 141980, Dubna, Moscow Region, Russia
}

\begin{abstract}
We discuss constraints on supersymmetric models arising from the relic density measurements of WMAP as well as from direct and precision measurements, LEP, $b \rightarrow s \gamma,(g-2)_{\mu}, B_{s} \rightarrow \mu^{+} \mu^{-}$. We consider mSUGRA models and their extensions with non-universal gaugino masses. We find, as commonly known, that the relic density pinpoints towards very specific regions of the mSUGRA models: coannihilation, focus and Heavy Higgs annihilation. The allowed regions widen significantly when varying the top quark mass. Introducing some non-universality in the gaugino masses significantly changes this conclusion as in specific non-universal models the relic density upper limit can be easily satisfied. This occurs with models where $M_{1}>M_{2}$ at the GUT scale when the LSP has a high wino component. Models where $M_{3}<M_{2}$ favours the Higgs annihilation channel in large regions of parameter space and at large $\tan \beta$ also favours the annihilation of neutralinos in gauge bosons pairs. We discuss also the potential of direct detection experiments to probe supersymmetric models and point out at the main consequences for colliders based on the mass spectrum. Our calculation of the relic density of neutralinos is based on micrOMEGAs and the SUSY spectrum is generated with SOFTSUSY.
\end{abstract}

LAPTH-1052/04

September 1, 2018 
Despite the lack of direct experimental evidence for supersymmetry, the minimal supersymmetric standard model (MSSM) remains one of the most attractive extensions of the standard model. One of the nice features of the MSSM is that it provides a natural cold dark matter candidate, the lightest supersymmetric particle (LSP). Recently, the WMAP satellite has measured precisely many cosmological parameters, among them the matter density, the baryonic density as well as the relic neutrino density [1, 2. From these measurements one can infer the relic density of cold dark matter, $\Omega h^{2}=.1126_{-.0181}^{+.0161}$ (at $2 \sigma$ ). This experimental measurement severely constrains the parameter space of supersymmetric models [3, 4, 5, 6]. This is particularly true in mSUGRA models where most of the analyses have been done so far. Although it has been argued that some realisations of mSUGRA are in fact quite generic to more general MSSM models so that it is sufficient to restrict to mSUGRA, it rests that most of the interesting scenarios that might occur in mSUGRA require a careful adjustment of parameters. For example, the Higgsino LSP. Moreover some important scenarios are never realised in mSUGRA like for example having a LSP which is dominantly wino. Equality of the gaugino masses at the GUT scale $\left(M_{1}^{\prime}=M_{2}^{\prime}\right)$ leads to $M_{1}^{\prime}=0.4 M_{2}^{\prime}$ at the weak scale thus to a LSP that is either mostly bino or a mixed bino/Higgsino state. In fact, in mSUGRA, most of the time, at least in what constitutes from the relic density point of view the bulk region, the LSP is an almost pure bino. The bulk region corresponds to the low $M_{0}-M_{1 / 2}$ region of parameter space of mSUGRA models [7, 8, 9]. Here $M_{0}\left(M_{1 / 2}\right)$ stands for the common scalar (gaugino) mass defined at the GUT scale. The recent cosmological data has almost ruled out this scenario [4, 5, 6, 10, This is because the main annihilation channel is into a pair of fermions and proceeds through the t-channel exchange of a right-handed slepton, the fermions with the largest hypercharge. The annihilation of a pure bino is not very efficient since the $\mathrm{U}(1)$ coupling, $g=e / c$, is small and weaker than a $\mathrm{SU}(2)$ coupling $g=e / s$. In order to bring down the relic density in the desired range one must appeal to specific mechanisms, either annihilation of neutralinos via s-channel Higgs exchange [1] or coannihilation of neutralinos with other sfermions [12]. Finally when the LSP has a significant Higgsino component, then annihilation into gauge bosons and/or coannihilation with heavier neutralino/chargino becomes very efficient [7], so much so that the relic density tends to be below the range of WMAP [13].

Several analyses have shown that at least one of these conditions is satisfied only for narrow strips in the parameter space of the mSUGRA model [3, 4, 15]:

- coannihilation with $\tilde{\tau}_{1}$ occur in the low $M_{0}$ region and requires $m_{\tilde{\tau}_{1}} \approx m_{\tilde{\chi}_{1}^{0}}$ (the coannihilation region),

- annihilation via a heavy scalar Higgs into a pair of b-quarks or $\tau$ leptons is possible at large $\tan \beta$ (the Higgs funnel region)

- one finds a neutralino with a high Higgsino content at large values of $M_{0}$ when the parameter $\mu$ becomes small (the focus point region). This occurs near the electroweak symmetry breaking (EWSB) border.

The strong correlation between the parameters are somewhat responsible for the rather contrived constraints coming from the relic density in mSUGRA models. However it was also pointed out that when taking into account the uncertainty in the input parameters, such as the top quark mass $m_{t}$, the allowed region widens significantly [10, 14]. This is particularly true both in the focus point region and in the heavy Higgs annihilation region. 
In the former, the value of the $\mu$ parameter, which drives the Higgsino nature of the LSP, is extremely sensitive to $m_{t}$ [15, 16. In the latter it is the heavy Higgs masses themselves that depend on $m_{t}$. In the coannihilation region, the top quark mass dependence shows up when applying the Higgs mass limit. The direct limit is somewhat relaxed with a large top quark mass, therefore we pay special attention to the $m_{t}$ dependence. We confirm qualitatively the results of previous analyses [3, 4, 5. although we differ in the position of the focus point and of the heavy Higgs funnel regions.

To go beyond the much contrived mSUGRA model while keeping a manageable number of free parameters, we introduce non-universality in the GUT-scale relation for gauginos. Such non-universality can be found in a variety of models that go beyond mSUGRA. For example, SUGRA models with non-minimal kinetic terms [17], superstring models with moduli-dominated or a mixture of moduli and dilaton fields and anomaly mediated SUSY breaking models [18, 19, 20, 21, 22, 23, all feature non-universal masses in the gaugino and/or scalar masses. Here we just introduce two free non-universality parameters relating the gaugino masses at the high scale, $r_{12}=M_{1} / M_{2}$ and $r_{32}=M_{3} / M_{2}$.

Basically in non-universal gaugino mass models one finds the same dominant channel for neutralino annihilation, however the parameter space for which annihilation into leptons, s-channel annihilation near a resonance, coannihilation or Higgsino/wino annihilation occurs and give a reasonable value for the relic density differs significantly from mSUGRA model. Most importantly, we do not have to carefully tune parameters to fall in the allowed regions. It is therefore important to explore the relic density as well as other constraints from precision measurements on these categories of models in order to assess the potential of both future colliders as well as direct/indirect searches for the LSP. For one, increasing the ratio $M_{1} / M_{2}$ provides a LSP with a much higher wino content. Then gaugino annihilation/coannihilation into gauge bosons becomes the dominant process 24]. Values for the relic density below the WMAP upper bound are found for large regions of the $M_{0}-M_{1 / 2}$ parameter space. On the other hand decreasing the $M_{1}^{\prime} / M_{2}^{\prime}$ ratio, one can more easily find light neutralinos that can annihilate via a s-channel $\mathrm{Z}$ or light Higgs pole provided $\mu$ is small enough. This was discussed in details in 25, 26, 27. A shift in the ratio $M_{3}^{\prime} / M_{2}^{\prime}$ also induces significant changes in the prediction for the relic density. Indeed, the parameter $M_{3}^{\prime}$ has a large impact on the spectrum calculation, driving the masses of all coloured particles but also changing the relation between $\mu$ and $M_{A}$, two parameters that are critical for the estimate of the relic density. Thus, decreasing $r_{32}$ introduces noticeable shifts in the region of parameter space where neutralinos annihilate via a heavy Higgs pole. In particular this annihilation channel can be relevant at low values of $\tan \beta$. Furthermore one finds much lighter squarks than in mSUGRA models favouring the coannihilation with squarks.

Although we focus mainly on the relic density constraint, we also take into account the experimental constraints set by direct searches at colliders as well as those coming from precision measurements, including $b \rightarrow s \gamma,(g-2)_{\mu}, B_{s} \rightarrow \mu^{+} \mu^{-}$. As concerns the limit from the muon anomalous magnetic moment, there have been many changes both in the theoretical estimates as well as improved experimental bounds in the last years [28. In particular, the standard model prediction depends on how the lowest-order hadronic contribution is extracted from the data, either using $e^{+} e^{-}$or $\tau$ data [29. We quote both the $e^{+} e^{-}$estimate or an average of all results. We think it would be overly conservative not to include at all this constraint. After all the upper allowed value for $\delta a_{\mu}$ has been stable over the many refinements of both theoretical and experimental results. 
When dealing with constraints on the mSUGRA parameters, one crucial step is the evaluation of the physical spectrum corresponding to a given mSUGRA model. This is based on the renormalization group equations (RGE) that specify the evolution of parameters from the high scale to the low scale as well as on the inclusion of radiative corrections to the physical masses of sparticles. This introduces some theoretical uncertainties in the prediction of the spectrum and can seriously affect the prediction of observables at low energies and in particular of the relic density. Here we will present results obtained with SOFTSUSY1.8.6 [30]. A detailed analysis of the difference between codes for the prediction of the relic density as well as a more detailed study of the theoretical uncertainties in the evaluation of the spectrum and their implications for the relic density will appear elsewhere [31, 32].

The relic density measurement is not the only way to probe supersymmetric dark matter in space, many experiments are underway and even more have been planned to directly search for dark matter. These experiments are effectively sensitive to either the spin independent (scalar) or spin dependent neutralino proton scattering cross section. The neutralino proton cross section proceeds through Z/Higgs or squark exchange. Thus a large cross section requires either light squarks and/or significant coupling with the Z/Higgs. In mSUGRA models this means that large cross sections are typically found in the focus point region. The current series of experiments have reached a sensitivity on the scalar cross section at the level $\sigma_{\tilde{\chi}_{1}^{0}-p} \approx 10^{-6} \mathrm{pb}$ [34. The only report of a positive signal by DAMA 35] has not been confirmed by others ${ }^{2}$. To really probe mSUGRA models however, one still has to wait for improved detectors such as CDMS2 or EdelweissII. CDMS2 has recently published much improved upper limits [36]. By the time one reaches ton-size detectors, such as ZeplinIV 37, Genius [38] and Xenon 39] a signal could be found for a significant fraction of the parameter space of the mSUGRA model. At the same time many detectors that are mostly sensitive to the spin dependent part of the neutralino nucleon cross section have started taking data, for example Naiad [0], Simple 41] and PICASSO 42]. Upgrades of these detectors are planned and eventually they will reach a sensitivity between $10^{-5}-10^{-4} \mathrm{pb}$.

Our calculation of the relic density is based on micrOMEGAs1.3 [43, 44, a code for the calculation of the relic density of dark matter which includes all annihilation and coannihilation channels. Although all cross sections are calculated exactly only at treelevel, some important loop effects are taken into account: loop corrections in masses as well as in vertices, loop corrections for the Higgs widths are also included. The pole masses and all mixing matrices are obtained from a code that calculates the supersymmetric spectrum. Additional SUSY corrections to the $h b \bar{b}$ vertices (the $\Delta m_{b}$ corrections ) are calculated independently. Although the radiative corrections to neutralinos, charginos and sleptons are rather small, they can play a crucial role in the case of coannihilation. Other constraints from precision measurements are included as well in the code: $b \rightarrow s \gamma$, $(g-2)_{\mu}, B_{s} \rightarrow \mu^{+} \mu^{-}$.

The paper is organized as follows: we first review the model parameters in section 1 and the direct and indirect constraints in section 2. In section 3 we present results in the mSUGRA scenario taking also into account uncertainties in the top quark mass. We first summarize the constraints form colliders and precision measurements before discussing at length the relic density constraints. As a first step towards a full analysis of the MSSM we

\footnotetext{
${ }^{2}$ This discrepancy might be explained using different velocity distributions for light dark matter particles 33 .
} 
then introduce non-universality in the gaugino sector in section 4 . The potential for both the spin-dependent/spin-independent direct detection experiments for the mSUGRA and non-universal mSUGRA models will be discussed in Section 5. Our results are summarized in the conclusion.

\section{Model parameters}

The parameters of the MSSM Lagrangian that are necessary for the calculation of the various processes are obtained using the renormalization group equations (RGE's) starting with the input parameters of mSUGRA models defined at the GUT scale, $M_{0}$ the scalar mass, $M_{1 / 2}$ the common gaugino mass, $A_{0}$ the common trilinear coupling, $\tan \beta$ the ratio of the Higgs vev's defined at the weak scale and $\operatorname{sgn}(\mu)$, the sign of the Higgs mixing parameter. The convention used are those of the SUSY Les Houches Accord [45], we only repeat here the convention for the neutralino mass matrix:

$\mathcal{M}_{\tilde{\chi}}=\left(\begin{array}{cccc}M_{1} & 0 & -M_{Z} \cos \beta \sin \theta_{W} & M_{Z} \sin \beta \sin \theta_{W} \\ 0 & M_{2} & M_{Z} \cos \beta \cos \theta_{W} & -M_{Z} \sin \beta \cos \theta_{W} \\ -M_{Z} \cos \beta \sin \theta_{W} & M_{Z} \cos \beta \cos \theta_{W} & 0 & -\mu \\ M_{Z} \sin \beta \sin \theta_{W} & -M_{Z} \sin \beta \cos \theta_{W} & -\mu & 0\end{array}\right)$,

Here $M_{i}$, the gaugino masses, as well as other parameters entering the matrix are defined at the electroweak symmetry breaking scale. The nature of the LSP, $\tilde{\chi}_{1}^{0}$, which is a linear combination of bino $\tilde{B}$, wino $\tilde{W}$ and the two Higgsino states $H_{1,2}$, is a crucial parameter in the evaluation of the relic density;

$$
\tilde{\chi}_{1}^{0}=N_{11} \tilde{B}+N_{12} \tilde{W}+N_{13} \tilde{H}_{1}+N_{14} \tilde{H}_{2}
$$

where $N$ is the neutralino mixing matrix. By inspection of the neutralino matrix one sees that if $M_{1}<<M_{2}, \mu$ the lightest neutralino is almost a pure bino, if $\mu<<M_{1}, M_{2}$ it becomes dominantly Higgsino and when $M_{2}<<M_{1}, \mu$ the neutralino is dominantly wino. In mSUGRA models, one always finds that $M_{1} \approx 0.4 M_{2}$ at the electroweak scale, moreover in most cases $M_{2}<\mu$. Thus the lightest neutralino is mainly a bino. This also leads to the usual mass relation between the LSP and the lightest chargino $m_{\tilde{\chi}_{1}^{0}} \approx .5 m_{\tilde{\chi}_{1}^{+}}$. Within the mSUGRA model, there also exists a possibility for a LSP with a much larger Higgsino component, this occurs in the focus point region at large $M_{0}$. There, the parameter $\mu$ decreases very rapidly until eventually $\mu^{2}<0$ and the EWSB cannot take place. When $\mu<M_{1}, M_{2}$, the mass of the LSP is driven by $\mu$ and one has $m_{\tilde{\chi}_{1}^{0}} \approx m_{\tilde{\chi}_{2}^{0}} \approx m_{\tilde{\chi}_{1}^{+}}$. The coupling of the neutralino to the $\mathrm{Z}$ depends on the Higgsino fraction and is therefore enhanced in the focus point region. The coupling of the neutralino to the light Higgs also requires some Higgsino component and is therefore also enhanced in the focus point region of mSUGRA.

The parameter $\mu$ is extracted from the minimization of the potential at the electroweak scale. At tree-level,

$$
\mu^{2}=\frac{m_{H_{1}}^{2}-m_{H_{2}}^{2} \tan ^{2} \beta}{\tan ^{2} \beta-1}-\frac{1}{2} M_{Z}^{2}
$$


where $m_{H_{1}}, m_{H_{2}}$ are the two soft scalar masses in the Higgs potential. Thus the value for $\mu$ strongly depends on the RG evolution of these scalar masses. In mSUGRA one finds typically large values for $\mu$ as compared to the gaugino masses. In more general models defined at the GUT scale one introduces new parameters at the GUT scale which can change the relation among parameters at the weak scale, it is then possible to obtain a LSP with a significant wino or Higgsino component.

In models with non-universal gaugino masses we introduce two extra parameters that characterize the amount of non-universality,

$$
r_{12}=\frac{\left.M_{1}\right|_{G U T}}{\left.M_{2}\right|_{G U T}} \quad r_{32}=\frac{\left.M_{3}\right|_{G U T}}{\left.M_{2}\right|_{G U T}}
$$

where $\left.M_{i}\right|_{G U T}=M_{i}^{\prime}$ are the gaugino masses defined at the GUT scale. We always define $M_{2}^{\prime}=M_{1 / 2}$.

Relaxing the gaugino masses universality, one can have models where the LSP can have a large wino component. For example, $r_{12}>1$, leads to a LSP that has a significant wino component. LEP data then implies that $m_{\tilde{\chi}_{1}^{0}}>M_{W}$ which in turn favours large annihilation cross sections for neutralinos into gauge bosons. This also implies that the chargino can be almost degenerate with the LSP, $m_{\tilde{\chi}_{1}^{0}} \approx m_{\tilde{\chi}_{1}^{+}}$, hence the important contribution of coannihilation channels.

The parameter $r_{32}$ has a strong influence on the weak scale parameters. Not only does the gaugino mass $M_{3}$ determine the coloured sector but it also enters indirectly the RGE equations for $M_{H_{1}}, M_{H_{2}}$. In particular, this implies that both $\mu$ and $M_{A}$ also depend on $M_{3}$ and can be significantly shifted as compared to the unified case. Taking all scalar masses equal at the GUT scale and with $\tan \beta=10, A_{0}=0$, we obtain an approximate solution for $\mu^{2}$ at the weak scale,

$$
\begin{aligned}
\mu^{2} \approx & -0.5 M_{Z}^{2}+c_{0} M_{0}^{2}+2.6 M_{3}^{\prime 2}-0.2 M_{2}^{\prime 2}+0.005 M_{1}^{\prime 2}+0.034 M_{3}^{\prime} M_{1}^{\prime} \\
& +0.22 M_{3}^{\prime} M_{2}^{\prime}+0.007 M_{2}^{\prime} M_{1}^{\prime}
\end{aligned}
$$

The coefficient $c_{0}$ depends critically on the top mass at the SUSY scale, for $m_{t}=175 \mathrm{GeV}$, $c_{0} \approx 0.11$. $c_{0}$ can get very small and even negative which defines the focus point regions where $\mu$ becomes very small. Clearly then because of the partial cancellation between the term in $M_{3}^{\prime}$ and the one in $M_{2}^{\prime}$, when $r_{32}<1, \mu$ is smaller than in the unified case. This once again leads to a LSP with a higher Higgsino component and more degenerate with the lightest chargino. The former implies more efficient annihilation, the latter more efficient coannihilation.

The pseudoscalar mass also depends strongly on $M_{3}$. An approximate solution to the RGE equations under the same condition as above leads to

$$
M_{A}^{2} \approx-M_{Z}^{2}+1.08 M_{0}^{2}+2.6 M_{3}^{\prime 2}+0.28 M_{2}^{\prime 2}+0.22 M_{2}^{\prime} M_{3}^{\prime}+0.03 M_{1}^{\prime 2} .
$$

When $r_{32}<1$, the pseudoscalar mass is lowered as compared to the universal case and one can find a pole in the neutralino annihilation channel even for an intermediate value of $\tan \beta$. For example, when $r_{32}=1 / 2, m_{\tilde{\chi}_{1}^{0}}^{2}=.4 M_{2}^{2}$ and $M_{0} \approx .7 M_{2}$, we find $M_{A} \approx 2 m_{\tilde{\chi}_{1}^{0}}$. In the universal case such a solution can be found only at large values of $\tan \beta$. This will open up new regions of parameter space where neutralino annihilation proceeds through an exchange of a Higgs in the s-channel. 
Introducing non-universal Higgs masses would in many ways reproduce the features of the non-universal gaugino masses scenarios in the sense that both $\mu$ and $M_{A}$ are directly related to the Higgs scalar masses at the EWSB scale. For example, lowering $M_{3}$ is equivalent to taking $M_{H_{1}}$ larger than other scalar masses. Then it is possible to obtain values for $\mu$ and $M_{A}$ that are lower than in the universal case leading to more efficient annihilation/coannihilation of neutralinos. We do not discuss these scenarios any further, they have been discussed in [46].

In this paper we do not use the approximate solutions Eq. 5, 6] which only serve as a guide, but obtain the electroweak scale parameters directly by solving completely the RGE's. This is done by a call to SOFTSUSY, a program that solves the RGE equations and also calculates the radiatively corrected superparticle masses and couplings. These higher-order corrections are essential to precisely evaluate the relic density both in the coannihilation regions where the exact mass splitting is a crucial parameter and near a s-channel resonance.

\section{Constraints on supersymmetric models: Direct lim- its, $\Omega h^{2},(g-2)_{\mu}, b \rightarrow s \gamma, B_{s} \rightarrow \mu^{+} \mu^{-}$}

\section{Direct limits from LEP}

For the chargino mass, we use the LEP2 bound, $m_{\chi}^{+}>103.5 \mathrm{GeV}$ except when there is near degeneracy with the lightest neutralino $(\Delta M=\mathcal{O}(1) \mathrm{GeV})$, then $m_{\chi}^{+}>91.9 \mathrm{GeV}$ 48.

For the neutralino sector we do not use directly the lower limit on the neutralino mass quoted by the LEP experiments, $m_{\tilde{\chi}_{1}^{0}}<59.6 \mathrm{GeV}$ since this value implicitly assumes universal gaugino masses at the GUT scale, $M_{1}=M_{2}$ [49]. Rather, we use in addition to the constraint on the chargino mass, the processes $e^{+} e^{-} \rightarrow \chi_{1}^{0} \chi_{2}^{0}, \chi_{1}^{0} \chi_{3}^{0}$. The associated production can somewhat improve on the constraints set from the chargino pair production in scenarios where $M_{1}<M_{2}$ [50, 26] in particular in the region with small $\mu$. In our scans, we implemented the upper limit from the L3 experiment on $\sigma\left(e^{+} e^{-} \rightarrow \tilde{\chi}_{1}^{0} \tilde{\chi}_{2}^{0}+\tilde{\chi}_{1}^{0} \tilde{\chi}_{3}^{0} \rightarrow\right.$ $\not \mu^{+} \mu^{-}$) [47. The radiative process $e^{+} e^{-} \rightarrow \tilde{\chi}_{1}^{0} \tilde{\chi}_{1}^{0} \gamma$ and the invisible width of the Z, $\Gamma_{Z_{i n v}}<3 \mathrm{MeV}$, can in principle also constrain the neutralino sector but these can play a role only in models with light neutralinos [26. Even then, present limits are such that these processes do not help much in reducing the allowed parameter space.

For selectrons, a limit of $m_{\tilde{e}_{R}}>99.4 \mathrm{GeV}$ can be set when $m_{\tilde{\chi}_{1}^{0}}>40 \mathrm{GeV}$ while for other charged leptons the lower limits of $m_{\tilde{\mu}}>96.5 \mathrm{GeV}$ and $m_{\tilde{\tau}}>92.5 \mathrm{GeV}$ apply [48].

In models where the pseudoscalar mass is heavy, the limit on the Higgs mass from LEP2, $m_{H}>114.4 \mathrm{GeV}$, applies. However we have imposed the limit $m_{H}>111 \mathrm{GeV}$ to allow for theoretical uncertainties. The Higgs mass is calculated by the code for the SUSY spectrum calculation, here SOFTSUSY1.8.6, and includes the two-loop corrections of Feynhiggs [51] as well as the two-loop yukawa corrections [52. In models with a light pseudoscalar the above LEP2 constraint is relaxed, the absolute bound $m_{H}, M_{A}>$ 91.6 $\mathrm{GeV}$ is obtained when $\sin (\alpha-\beta) \approx 0$ [53, 54]. 


\section{Relic density of neutralinos $\Omega h^{2}$}

The supersymmetric models with a stable neutralino must be consistent with at least the upper limit on the amount of cold dark matter. We consider both the $2 \sigma$ limit from WMAP,

$$
0.094<\Omega h^{2}<0.129
$$

as well as only the upper limit since there could be some other contribution to the cold dark matter. Our calculations of the relic density is based on micrOMEGAs1.3, a program that calculates the relic density in the MSSM including all possible coannihilation channels [44, 43. Within the context of a model defined at the GUT scale such as universal/nonuniversal SUGRA that we consider here, we first need to calculate the MSSM parameters. To do this we use a renormalization group evolution code, SOFTSUSY to evaluate the supersymmetric mass spectrum as well as the mixing matrix elements following the convention of the SUSY Les Houches Accord [45. These parameters are then used together with $\tan \beta$ and $\mu$ to calculate all cross sections for annihilation of neutralinos or coannihilation. Although the mass and mixing parameters include one-loop corrections, this procedure is of course not a complete one-loop computation of the relic density. However the most important one-loop effects are taken into account. In particular the mass difference between the NLSP and the LSP are computed correctly. This mass difference is crucial in evaluating the relic density in the coannihilation region. Indeed in computing the effective annihilation cross section, coannihilation processes are suppressed by a Boltzmann factor $\propto \exp ^{-\Delta M / T_{f}}$ where $\Delta M$ is the mass difference between the NLSP and the LSP and $T_{f}$ the decoupling temperature $\left(T_{f} \approx M_{L S P} / 25\right)$. Then even a small shift in the masses can reflect significantly on the relic density. For the Higgs width the running of the b-quark mass including three loop corrections as well as the $\Delta m_{b}$ corrections are included [55].

Muon anomalous magnetic moment $(\mathrm{g}-2)_{\mu}$

The latest experimental data on the $g-2$ measurement using $\mu^{-}$28], brings the average to

$$
a_{\mu}^{\text {exp. }}=11659208 \pm 6 \times 10^{-10}
$$

The quantity $a_{\mu}$ includes both electroweak and hadronic contributions and is still subject to large theoretical errors,

$$
a_{\mu}^{\mathrm{theo.}}=a_{\mu}^{\mathrm{QED}}+a_{\mu}^{\mathrm{weak}}+a_{\mu}^{\mathrm{HAD}}+a_{\mu}^{\mathrm{LBL}}+a_{\mu}^{\mathrm{HAD} / \mathrm{NLO}} .
$$

The largest uncertainty in the calculation of $a_{\mu}$ arises from the lowest order hadronic contribution. We take an average of four recent estimates: the value reported by Jegerlehner $a_{\mu}^{\text {had }}=(6889 \pm 58) \times 10^{-11}$ [56] that includes BES and CMD-2, the value extracted from inclusive data by Teubner et al. $a_{\mu}^{\text {had }}=(6831 \pm 59 \pm 20) \times 10^{-11}$ [57], the latest estimate by Davier et al. based on $e^{+} e^{-}$data $a_{\mu}^{\text {had }}=\left(6963 \pm 62_{\text {exp }} \pm 36_{t h}\right) \times 10^{-11}$ [29] and finally the result of Yndurain, $a_{\mu}^{\text {had }}=\left(6935 \pm 50_{\text {exp }} \pm 10 \pm 5_{t h}\right) \times 10^{-11}[58$. The latter two authors also report another estimate that is based on $\tau^{+} \tau^{-}$data. The values extracted from $\tau$ data are consistent with each other but differ from the values obtained from $e^{+} e^{-}[29$. For the hadronic light by light contribution we take $a_{\mu}^{L B L}=(+80 \pm 40) \times 10^{-11}$ [59] which is based on the calculation of Ref. 60, 61, 62, and includes a large theoretical error.

We get, after averaging the different hadronic contributions from $e^{+} e^{-}$data alone,

$$
\delta a_{\mu}=a_{\mu}^{\text {exp. }}-a_{\mu}^{\text {theo. }}=\left(33.8 \pm 6_{\mid \text {exp. }} \pm 11.5_{\mid \text {theo. }}\right) \times 10^{-10}
$$


while including also the $\tau$ estimate we get

$$
\delta a_{\mu}=a_{\mu}^{\text {exp. }}-a_{\mu}^{\text {theo. }}=\left(28.5 \pm 6_{\mid \text {exp. }} \pm 11.5_{\mid \text {theo. }}\right) \times 10^{-10}
$$

Adding the errors in quadrature leads to the $2 \sigma$ allowed range, for the $e^{+} e^{-}$data alone,

$$
7.8<\delta a_{\mu} \times 10^{10}<59.7
$$

while including $\tau$ data in the average, the value moves closer to the standard model,

$$
2.5<\delta a_{\mu} \times 10^{10}<54.4
$$

Note that our central value is slightly higher than the one reported by Davier [29] as we have averaged all estimates of the lowest-order hadronic contribution. We will in general only show contour levels for $\delta a_{\mu}$. We remark that in the last couple of years there has been many improvements to the computation of the standard contribution to $(g-2)_{\mu}$, and the allowed area has shifted significantly. Still the upper bound has been rather stable and therefore should be considered as a conservative constraint on the supersymmetric model.

$$
\mathbf{b} \rightarrow \mathbf{s} \gamma
$$

Our calculation of the branching ratio for $b \rightarrow s \gamma$ takes into account next-to-leading (NLO) order terms, bremstrahlung and some non-perturbative effects [63, 64, 67]. Our standard model value (with scale parameters set at $m_{b}$ ), gives $\operatorname{Br}(b \rightarrow s \gamma)=3.72 \times 10^{-4}$ while the scale and other parameter uncertainty $\left(\alpha_{s}\right.$, CKM ...) are about $10 \%$ 43. The Wilson coefficients for the charged Higgs contribution are evaluated at NLO 65] while SUSY contributions at leading-order are included as well as the important large $\tan \beta$ effects 66]

The experimental value for the branching ratio is extracted from an average of the Babar 68, CLEO 69] BELLE[70] and ALEPH [1] measurements

$$
\operatorname{Br}(b \rightarrow s \gamma)=3.34 \pm .38 \times 10^{-4}
$$

and assumes a fully correlated theoretical error 72$]$.

We require that after allowing for the (scale) uncertainty in the theory calculation the result must be within $2 \sigma$ of the experimental result, Eq. 12 . Since the theory uncertainty is roughly constant over the SUSY parameter space we have allowed for a conservative fixed uncertainty of $10 \%$ independently of the SUSY parameters. Thus in effect we require the theory prediction to fall within the range

$$
2.25<\operatorname{Br}(b \rightarrow s \gamma) \times 10^{4}<4.43
$$

$\mathbf{B}_{\mathbf{s}} \rightarrow \mu^{+} \mu^{-}$

The CDF experiment at Fermilab has obtained an upper bound on the branching ratio $\operatorname{Br}\left(B_{s} \rightarrow \mu^{+} \mu^{-}\right)<9 \times 10^{-6}[73]$ and should be able to reach $\operatorname{Br}\left(B_{s} \rightarrow \mu^{+} \mu^{-}\right)<2 \times 10^{-7}$ in RunII. In the SM, this branching ratio is expected to be very small $\left(\approx 3 \times 10^{-9}\right)$. In the MSSM, SUSY loop contributions due to chargino, sneutrino, stop and Higgs exchange can significantly increase this branching ratio. In particular, the amplitude for Higgs mediated decays goes as $\tan \beta^{3}$ and orders of magnitude increase above the SM value are expected for large $\tan \beta$. Our calculation is based on [74] and agrees with [75]. The $\Delta m_{b}$ effects relevant for high $\tan \beta$ are taken into account. 


\section{Results in mSUGRA}

We have performed scans over the mSUGRA parameter space using the code SOFTSUSY1.8.6. We first present results in the $M_{0}-M_{1 / 2}$ plane for different choices of $A_{0}$ and $\tan \beta$ and with fixed values of the top quark mass. We discuss constraints from precision measurements before concentrating on the relic density constraints. We consider only models with $\mu>0$ as they are the ones favoured by the $(g-2)_{\mu}$ measurement [76]. We note from the onset that there are theoretical uncertainties in the evaluation of the spectrum of the MSSM within mSUGRA models 16. These can have a large impact on the predictions for the relic density [31. Here we fix $m_{b}\left(m_{b}\right)=4.23 \mathrm{GeV}$ and choose values of $m_{t}$ within the $1 \sigma$ range, $m_{t}=174.3 \pm 5.1 \mathrm{GeV}$ [7]. ${ }^{3}$

\subsection{Precision measurements and direct limits}

The mass of the lightest Higgs is basically set by the top and the stop quark sectors. Heavier masses are found in regions with large $\tan \beta$ and large mixing in the stop sector. One expects a sensitivity to the input value of $m_{t}$, as well as to the value of $A_{0}$ which sets the parameter $A_{t}$ that drives the mixing among the stop quarks. Large mass splitting between the stops is difficult to find in the low $M_{0}-M_{1 / 2}$ region of parameter space. In this region the Higgs tends to be too light as seen from the contours of constant Higgs masses $m_{h}=111,114 \mathrm{GeV}$ in Fig. 1. An increase in the top quark mass also increases the Higgs mass. This has particularly a large impact on models with small values of $\tan \beta$. For example, for $\tan \beta=5(10)$ and for small $M_{0}$, the allowed region shifts from $M_{1 / 2}>417(271) \mathrm{GeV}$ for $m_{t}=175 \mathrm{GeV}$ to $M_{1 / 2}>332(230) \mathrm{GeV}$ for $m_{t}=179$ $\mathrm{GeV}$. For $\tan \beta=10$, the Higgs mass limit alone translates into a lower bound on the LSP $m_{\tilde{\chi}_{1}^{0}}>105(87.4) \mathrm{GeV}$ as well as on the lightest chargino $m_{\tilde{\chi}_{1}^{+}}>194(160) \mathrm{GeV}$ respectively. Of course lighter charginos, in fact down to the direct limit set by LEP, are allowed for larger $M_{0}$ which correspond to heavier sleptons, but these will in general not be relevant from the relic density point of view as we will discuss next. A decrease in the top quark mass conversely tightens the constraint on $M_{1 / 2}$, for example the $m_{t}=172 \mathrm{GeV}$ and $m_{h}=111 \mathrm{GeV}$ contour roughly coincides with the $m_{t}=179 \mathrm{GeV}, m_{h}=114 \mathrm{GeV}$ contour of Fig. 1a. At large $\tan \beta$, the Higgs mass increases as well, so that the constraint on both $M_{0}$ and $M_{1 / 2}$ is relaxed.

The shift in the Higgs mass contours as one varies $\left|A_{0}\right|<2000 \mathrm{GeV}$ is quite significant as displayed in Fig. 1 b. First note that imposing $A_{0}=0$ in mSUGRA, leads to a negative value for $A_{t}$ at the electroweak scale. Since the dominant correction to the Higgs mass depends on the mixing in the stop quark sector, $\propto A_{t}+\mu / \tan \beta$, larger Higgs masses will be found for $A_{0}<0$ where $\left|A_{t}\right|$ is large. Indeed in Fig. 1 p, one sees that with $A_{0}=-1 \mathrm{TeV}$, the Higgs mass increases loosening the constraints on the low $M_{0}-M_{1 / 2}$ corner of parameter space for $\tan \beta=10$. Already at $\tan \beta=35$, one finds $m_{h}>111 \mathrm{GeV}$ over the full parameter space. Conversely, constraints are tightened for $A_{0}=2 \mathrm{TeV}$. Note that the region where one can find a consistent solution to the RGE's depends on $m_{t}, \tan \beta$ and on the value of $A_{0}$. Hence some of the contours stop abruptly in Fig 1 when one meets a non-physical region. Furthermore, the region where the LSP is neutral also shifts with

\footnotetext{
${ }^{3}$ New results from the Tevatron indicate a higher value for the top quark mass $m_{t}=178 \pm 4.3[80$. The new central value falls within the range we have considered.
} 

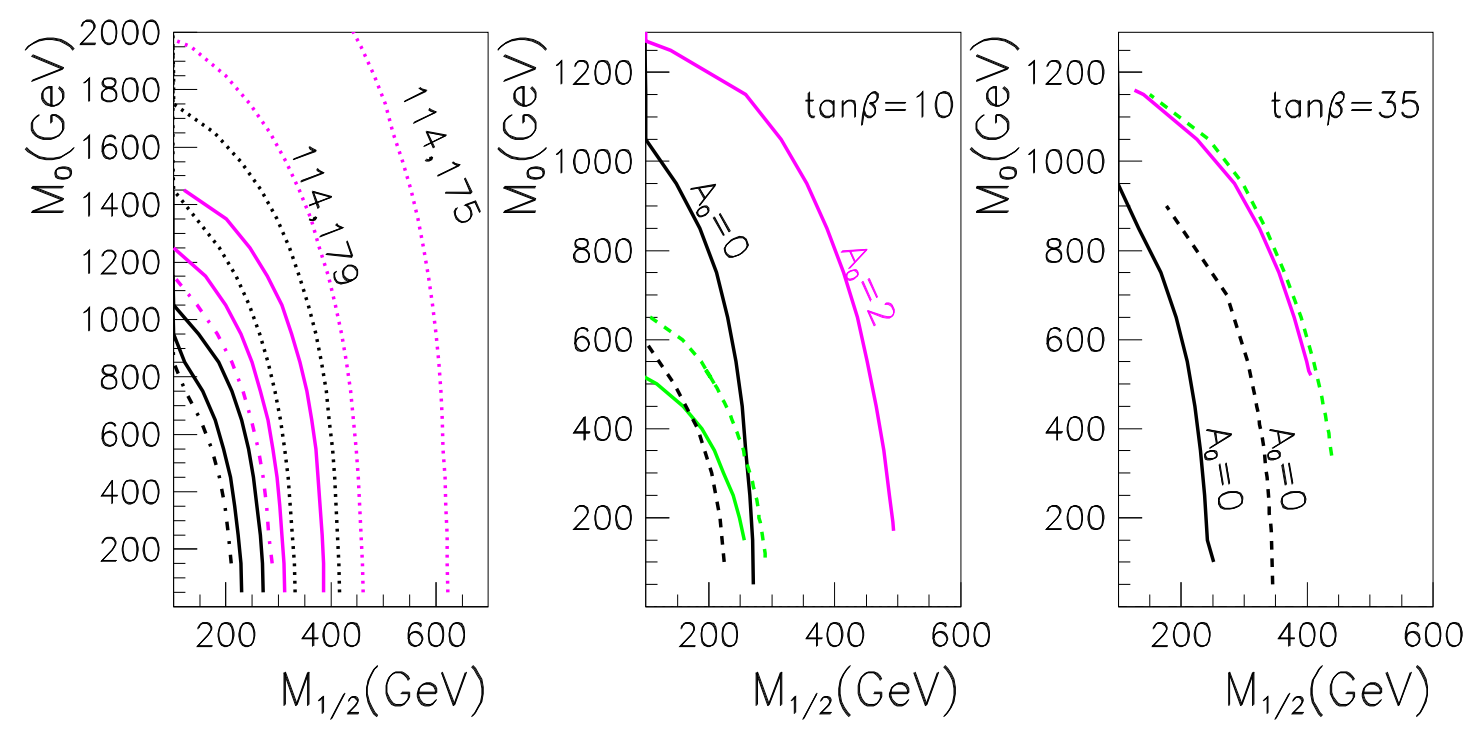

Figure 1: a) Contours of $m_{h}=111 \mathrm{GeV}$ (black) and $m_{h}=114 \mathrm{GeV}$ (pink/grey) in the $M_{0}-M_{1 / 2}$ plane for $\mu>0, A_{0}=0$ and for $\tan \beta=5$ (dot), $\tan \beta=10$ (full), $\tan \beta=35$ (dot-dash). Both contours for $m_{t}=175 \mathrm{GeV}$ and $m_{t}=179 \mathrm{GeV}$ are displayed. For each set of parameters, the contour for the heavier $m_{t}$ is on the left. For $\tan \beta=35$, only the contour for $m_{t}=175 \mathrm{GeV}$ is displayed. Some $m_{h}, m_{t}$ values are attached to the contours for easy reference. b) Effect of the trilinear mixing for $\tan \beta=10, A_{0}=0$ (black) $A_{0}=-1 \mathrm{TeV}$ (green/light grey), $A_{0}=2 \mathrm{TeV}$ (pink) on the $m_{h}=111$ GeV contours (full) and $\operatorname{Br}(b \rightarrow s \gamma)=2.2510^{-4}$ contours (dash). c) Contours for $m_{h}=111 \mathrm{GeV}$ (full), $\tan \beta=35$ with $A_{0}=0$ (black), $A_{0}=2 \mathrm{TeV}$ (pink) and for $\operatorname{Br}(b \rightarrow s \gamma)=2.2510^{-4}$ (dash) with $A_{0}=0$ (black), $A_{0}=-1 T e V$ (green/light grey). 
$\tan \beta, m_{t}$ and/or $A_{0}$ mainly because of the mixing in the $\tilde{\tau}$ sector as will be discussed in the next section.

The rare processes $b \rightarrow s \gamma, B_{s} \rightarrow \mu^{+} \mu^{-}$as well as $\delta a_{\mu}$ all provide large supersymmetric corrections at large values of $\tan \beta$ especially when the sfermions are light. The branching ratio for $b \rightarrow s \gamma$ depends mostly on the squark and gaugino/higgsino sector as well as on the charged Higgs. Even heavy squarks do not completely decouple and one can get substantial corrections to the SM branching ratio. The chargino exchange diagram gives a negative contribution relative to the SM one (for $\mu>0$ ) and the branching ratio for $b \rightarrow s \gamma$ drops below the allowed band in the low $M_{0}-M_{1 / 2}$ region. Furthermore there is a strong $A_{t}$ dependence from the mixing in the stop sector. For $\tan \beta=10$ and $m_{t}=175 \mathrm{GeV}$, the $b \rightarrow s \gamma$ constraint is not as severe as the constraint from the Higgs mass as long as $A_{0} \geq 0$, Fig. 1. For $A_{0}=2 \mathrm{TeV}$, the $b \rightarrow s \gamma$ constraint is satisfied over the full parameter space. For a large negative value for the trilinear coupling, $A_{0}=-1 \mathrm{TeV}$, the $b \rightarrow s \gamma$ constraint dominates. As one increases $\tan \beta$, for example $\tan \beta=35$, the dependence on $A_{0}$ is even more striking both for the Higgs mass and for the $b \rightarrow s \gamma$ branching ratio, especially that the shifts on the allowed parameter space work in opposite directions. For $A_{0}=2 \mathrm{TeV}$, the Higgs mass constraint is the most severe since the $b \rightarrow s \gamma$ plays no role while for $A_{0}=-1 \mathrm{TeV}$, the $b \rightarrow s \gamma$ constrains values of $M_{1 / 2}$ all the way to $450 \mathrm{GeV}$ whereas the Higgs mass constraint has no impact, see Fig. 1b. For $A_{0}=0$, it is the $b \rightarrow s \gamma$ constraint that dominates. In the region of light sleptons which will be relevant from the relic density point of view, the lower bound on $M_{1 / 2}=345(435) \mathrm{GeV}$ for $\tan \beta=35$ and $A_{0}=0(-1) \mathrm{TeV}$ implies $m_{\tilde{\chi}_{1}^{0}}>139(181) \mathrm{GeV}$ and $m_{\tilde{\chi}_{1}^{+}}>261(347) \mathrm{GeV}$. As one further increases the value of $\tan \beta$ one retains the same features: only the size of the forbidden region at low $M_{0}-M_{1 / 2}$ increases.

The branching ratio for $B_{s} \rightarrow \mu^{+} \mu^{-}$is predicted to be much below current experimental limits at low $\tan \beta$, however it increases rapidly with $\tan \beta$. There the chargino/stop exchange contribution dominates and it is largest in the low $M_{0}-M_{1 / 2}$ region[79]. This constraint depends somewhat on the top quark mass which influences the spectrum in the stop sector. The branching ratio is also dependent on the value of the mixing in the stop sector. Negative values of $A_{0}$, which as we have discussed above decrease $\left|A_{t}\right|$, strengthen the constraint. Eventually $B_{s} \rightarrow \mu^{+} \mu^{-}$will become an important constraint, this occurs typically for larger values of $\tan \beta$ that we have considered [79]. Among the cases studied here it is only for $\tan \beta=50$ that in RunII at Tevatron one could put restrictions on the allowed parameter space, for example for the case $A_{0}=-1000 \mathrm{GeV}$ shown in Fig 8

As for $(g-2)_{\mu}$, the allowed parameter space depends critically on how much of a deviation from the standard model one imposes. A significant contribution to $\delta a_{\mu}$ is obtained with light charginos and light sleptons. The upper limit on $\delta a_{\mu}$ then rules out the lower left hand corner of the $M_{0}-M_{1 / 2}$ plane. Larger deviations from the standard model are predicted as one increases $\tan \beta$ but this constraint does not play an important role once the limit on the Higgs mass is taken into account. On the other hand, requiring a non zero deviation from the standard model implies not so heavy sleptons and neutralinos, thus constraining the large $M_{0}-M_{1 / 2}$ region of parameter space as well. This region is more important at small values of $\tan \beta$ where, as just mentioned, one tends to get predictions consistent with the standard model, as displayed in Fig 2 for the contour $\delta a_{\mu}=5 . \times 10^{-10}$. Introducing a negative value for $A_{0}$ does not have a large impact on the prediction for $\delta a_{\mu}$ as it is the combination $A_{\mu}+\mu \tan \beta$ that comes into play in the smuon mixing. For intermediate to large $\tan \beta$, the mixing is dominated by $\mu \tan \beta$. 
To summarize the collider constraints in mSUGRA type models, the mass of the Higgs is the main constraint in the low $M_{0}-M_{1 / 2}$ region for values of $\tan \beta<35$ and is dependent both on the value of the top quark mass and the trilinear mixing. For $\tan \beta \geq 35$, the $b \rightarrow s \gamma$ constraint takes over, at least for $A_{0}$ not too large or for $A_{0}<0$. If one takes into account the lower limit from $\delta a_{\mu}$ one ends up with an allowed band in the $M_{0}-M_{1 / 2}$ plane. This band moves towards higher values of $M_{1 / 2}$ and increases in size with increasing $\tan \beta$. We stress again that the upper limit on $\delta a_{\mu}$ is rather conservative but the lower limit is still subject to debate. In the following we will display the contours for fixed values of $\delta a_{\mu}$.

\subsection{Relic density}

Within the context of mSUGRA models, one needs a special tuning of parameters to be in agreement with the very precise measurement on the relic density by WMAP. We first remark that the so-called bulk region (at low $M_{1 / 2}$ and $M_{0}$ ) that used to be one of the favoured regions [7, 8] has considerably shrunk. In the bulk region the LSP is almost a pure bino. As such, the LSP couples mostly to fermion/sfermions with the largest hypercharge, that is the right-handed sleptons. The dominant annihilation channel for neutralino annihilation is into a pair of fermions via the t-channel exchange of a righthanded sfermion. This annihilation process is not efficient enough to satisfy the new tight upper limit on the relic density of dark matter. One needs some additional contribution from the $\tilde{\tau}_{1}$-coannihilation or Higgs exchange channels. In fact, the region at low $M_{0}-M_{1 / 2}$ is only allowed at large $\tan \beta$ because there one gets extra contribution from s-channel Higgs exchange as well as from coannihilation. As we have just discussed the low $M_{0}-M_{1 / 2}$ region is also constrained from the Higgs mass limit at least for intermediate $\tan \beta$ and by the measurement of $b \rightarrow s \gamma$ for $\tan \beta>35$. We also remark that the top quark mass plays an important role in determining the relic density constraint in mSUGRA models. In some cases the special tuning of parameters required to satisfy the relic density constraint can only be obtained for specific values of the top quark mass. For example two of the preferred regions, the focus point region and the heavy Higgs funnel regions, are very sensitive, through the renormalization group equations, to the value of the running top quarks mass. We first discuss in detail the case $m_{t}=175 \mathrm{GeV}$ before letting the mass vary within the $1 \sigma$ range.

\subsection{1 $m_{t}=175 \mathrm{GeV}$}

For any value of $\tan \beta$, the regions consistent with the WMAP measurement correspond, for a fixed value of $m_{t}$, to thin strips in parameter space. The WMAP allowed region corresponds to the very narrow area between the contours $\Omega h^{2}=.094$ and $\Omega h^{2}=.129$, see for example Fig. 2 for $m_{t}=175 \mathrm{GeV}$. We are then left mainly with three regions: $\tilde{\tau}_{1}$-coannihilation, focus point and Higgs funnel. Although we will generally refer to the Higgs funnel region as the region where neutralino annihilation proceeds through the schannel exchange of a heavy Higgs scalar, we note that there is also a small region where light neutralinos annihilate efficiently near the light Higgs resonance. For example, this can be seen as the narrow vertical strip along the LEP2 limit on the chargino mass near $M_{1 / 2}=150 \mathrm{GeV}$, in Fig. [2 for $\tan \beta=10$.

In mSUGRA, the $\tilde{\tau}_{1}$-coannihilation region designates the region at low $M_{0}$, where the 

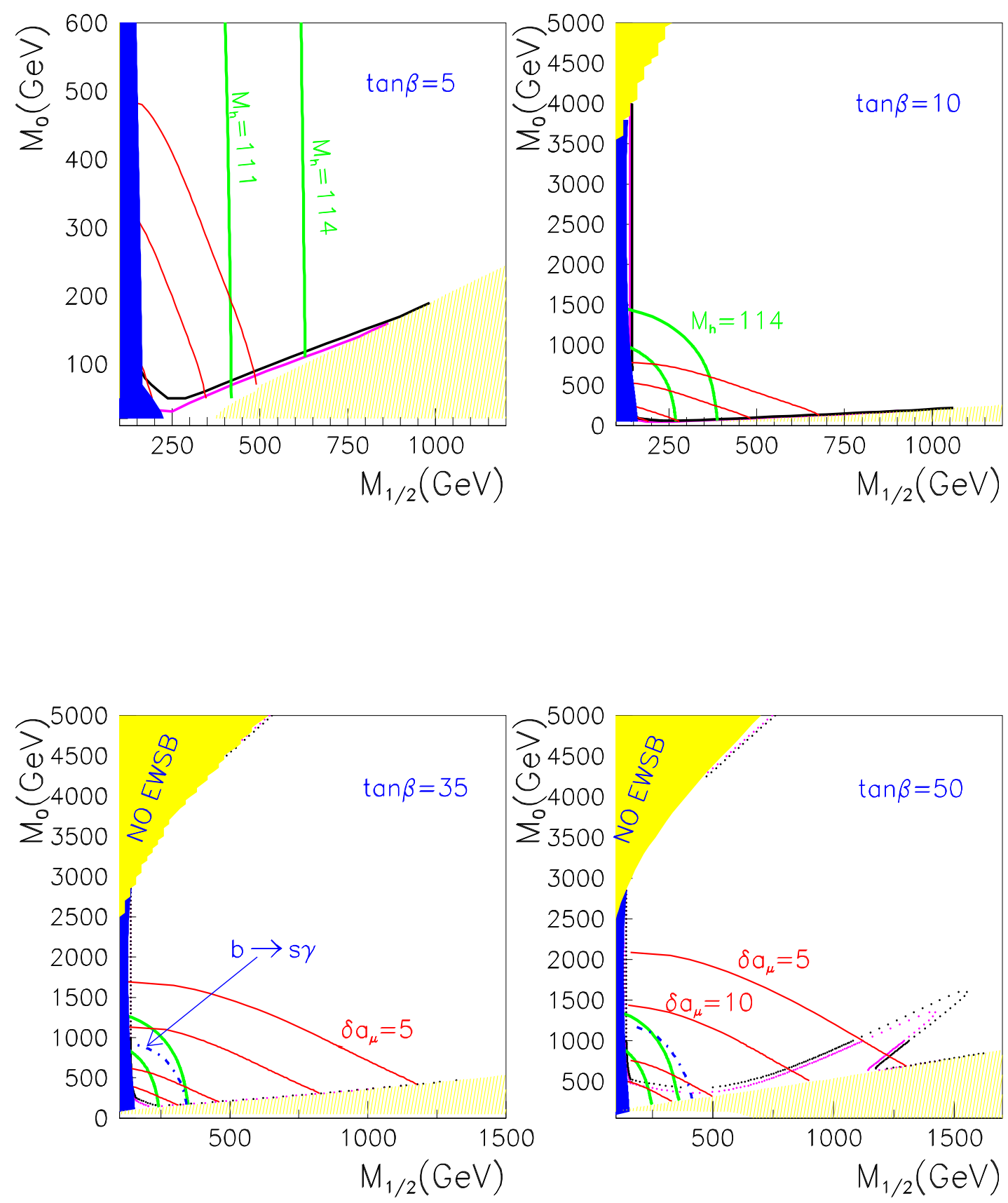

Figure 2: Allowed regions in the $M_{0}-M_{1 / 2}$ plane for $\tan \beta=5,10,35,50, A_{0}=0, \mu>0$ and $m_{t}=175 \mathrm{GeV}$. Contours for $m_{h}=111,114 \mathrm{GeV}$ (green) $\delta a_{\mu}=5,10,30,60 \times 10^{-10}$ (red) (top to bottom) and for $\Omega h^{2}=.129$ (.094) (black/pink dots). The contour $b \rightarrow s \gamma=$ $2.25 \times 10^{-4}$ (dash-dot/blue) is also displayed for $\tan \beta=35,50$. The region to the left of this contour is excluded. The LEP direct limits exclude the region delimitated by the blue vertical band. The regions between the $\Omega h^{2}=.094$ contour and the theoretically excluded regions (yellow/light grey) have a value for the relic density below the WMAP bound. 
lightest $\tilde{\tau}_{1}$ is the NLSP. As the relic density is very sensitive to the NLSP-LSP mass difference, the proper mass degeneracy for a relic density consistent with WMAP corresponds to a very narrow strip in the $M_{0}-M_{1 / 2}$ plane, see Fig. 2. Naturally this strip lies slightly above the forbidden region where $m_{\tilde{\tau}_{1}}<m_{\tilde{\chi}_{1}^{0}}$. This strong dependence on the mass difference also means that it is crucial to properly determine the pole masses from the running $\overline{D R}$ masses. In the coannihilation region, the main channels are $\tilde{\chi}_{1}^{0} \tilde{\tau}_{1} \rightarrow \tau \gamma$ and $\tilde{\tau}_{1} \tilde{\tau}_{1} \rightarrow \tau \bar{\tau}$. As one moves towards degenerate $\tilde{\tau} / \tilde{\chi}_{1}^{0}$, the relic density eventually drops below the WMAP range. Typically a value of the relic density within the WMAP range requires a mass difference $\Delta M_{\tilde{\tau} \tilde{\chi}_{1}^{0}} \approx 5-15 \mathrm{GeV}$ for $m_{\tilde{\chi}_{1}^{0}} \approx \mathcal{O}(100) \mathrm{GeV}$ while a smaller mass splitting is required for heavier neutralinos (near degeneracy is required for $m_{\tilde{\chi}_{1}^{0}}=400 \mathrm{GeV}$ ). Fig. 3 shows the relic density versus the mass difference for $\tan \beta=10,35$ as well as the mass difference as a function of the neutralino mass. At $\tan \beta=35$, one can afford a larger mass splitting as there is a larger contribution from the s-channel Higgs exchange to neutralino annihilation into fermions as well as an important contribution to $\tilde{\chi}_{1}^{0} \tilde{\tau} \rightarrow \tau h$. The latter contains a term proportionnal to the sfermion mixing. The mass difference between the LSP and the sleptons of the first two generations can be much larger and ranges from $10-20 \mathrm{GeV}$ for lower values of $\tan \beta$ up to $100 \mathrm{GeV}$ when $\tan \beta \geq 35$. There, the contribution from coannihilation processes with first generation sleptons become irrelevant ${ }^{4}$. Eventually, as the neutralino mass increases the coannihilation cross sections also become too small and one obtains an upper limit on the LSP mass. When combining this upper limit with the constraint imposed by the Higgs mass as well as from $b \rightarrow s \gamma$, we find the range of allowed values for $\tan \beta=10(35), 270(342) \mathrm{GeV}<M_{1 / 2}<1050(1250) \mathrm{GeV}$ corresponding to neutralinos of $105(138) \mathrm{GeV}<m_{\tilde{\chi}_{1}^{0}}<448(540) \mathrm{GeV}$.

The Higgs annihilation region includes both a light Higgs annihilation at low $M_{1 / 2}$ and a new region which appears only for large $\tan \beta$. The former occurs only for the LSP mass very close to $M_{h} / 2$ since the light Higgs has a very narrow width. The latter appears at moderate $M_{0}-M_{1 / 2}$ values and corresponds to the annihilation of neutralinos via the s-channel exchange of a heavy Higgs. The main channels are $\tilde{\chi}_{1}^{0} \tilde{\chi}_{1}^{0} \rightarrow b \bar{b}, \tau \bar{\tau}$ corresponding to the preferred decay channels of the Heavy Higgs. The crucial physical parameter is the mass difference between $M_{A}$ and $2 m_{\tilde{\chi}_{1}^{0}}$ relative to the pseudoscalar width, hence the importance of including higher-order corrections for the width [78]. Note that for very heavy scalars the width can be quite substantial, $\mathcal{O}(30) \mathrm{GeV}$. In Fig $\mathbb{4}$, the mass difference $\Delta M_{A \tilde{\chi}_{1}^{0}}=M_{A}-2 m_{\tilde{\chi}_{1}^{0}}$ relative to the width, $\Gamma_{A}$, is displayed as function of $M_{1 / 2}$ for $\tan \beta=50$. Here all constraints are applied. Models that fit within the WMAP range are roughly within $\Delta M_{A \tilde{\chi}_{1}^{0}}<2 \Gamma_{A}$ except in the low $M_{1 / 2}$ region. There the coannihilation region merges with the Higgs funnel region. Even though the coannihilation channels never dominate for these large values of $\tan \beta$ the contribution of the light sleptons to neutralino pair annihilation is sufficient to get an acceptable value for $\Omega h^{2}$. In fact, nearly degenerate $\tilde{\tau} / \tilde{\chi}_{1}^{0}$ will lead to a very low value for the relic density. Due to the heavy Higgs annihilation channel, neutralinos as heavy as $650 \mathrm{GeV}$ can give reasonable values for the relic density. This type of model could be very difficult to hunt at the LHC as it features a rather heavy spectrum. Indeed at the tip of the Higgs funnel, one finds all squarks and the gluino in the $2.5-3.2 \mathrm{TeV}$ range. Note that the position of the Higgs funnel is sensitive to the standard model input value used in the RGE codes, in particular $m_{b}\left(m_{b}\right)$ [83] as well as the top quark mass. Indeed the soft scalar masses in the Higgs potential,

\footnotetext{
${ }^{4}$ Note that the small mass difference between the stau and the LSP raises the question of detectability of a nearly degenerate $\tilde{\tau}$ lepton as well as the feasability of a precise measurement of its mass at a collider. For recent studies within the context of a linear collider see [81, 82].
} 

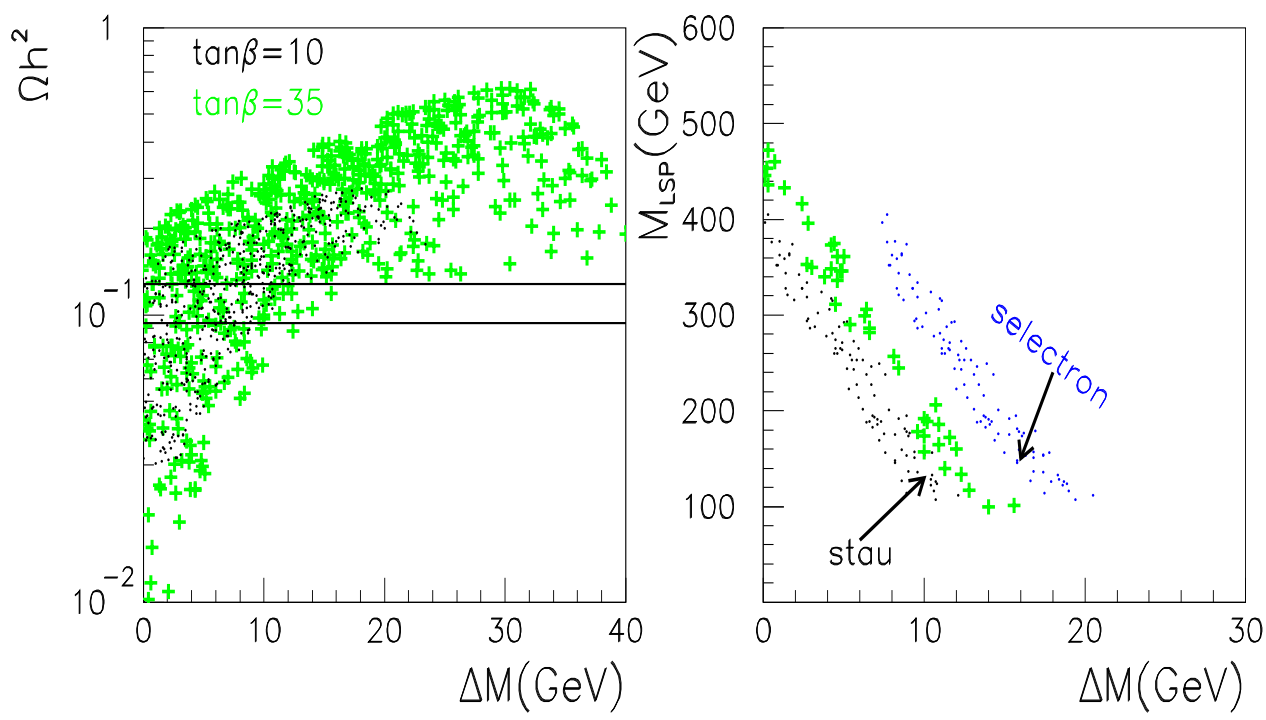

Figure 3: a) $\Omega h^{2}$ as a function of the mass difference $\Delta M_{\tilde{\tau} \tilde{\chi}_{1}^{0}}$ in the coannihilation region for $\tan \beta=10$ (black dots) and $\tan \beta=35$ (green crosses). b) For models where $\Omega h^{2}$ is within the WMAP range, $m_{\tilde{\chi}_{1}^{0}}$ as a function of the mass difference $\Delta M_{\tilde{\tau} \tilde{\chi}_{1}^{0}}$ for $\tan \beta=10$ (black dots) and $\tan \beta=35$ (green crosses) and as a function of the mass difference $\Delta M_{\tilde{e} \tilde{\chi}_{1}^{0}}$ for $\tan \beta=10$ (blue dots).

$m_{H_{1}}\left(m_{H_{2}}\right)$, from which one evaluates the mass of the pseudoscalar at the EWSB scale are very sensitive to the bottom (top) Yukawa's [16].

The last allowed region is the focus point region, this region is found at high values of $M_{0}$ where the value of $\mu$ drops rapidly. When $\mu<M_{1}, M_{2}$, the LSP has a significant Higgsino fraction. Furthermore the $\operatorname{NLSP}\left(\tilde{\chi}_{2}^{0}\right.$ and/or $\left.\tilde{\chi}_{1}^{ \pm}\right)$are also mostly Higgsino and are not much heavier than the LSP. Thus the neutralinos/charginos coannihilation channels are favoured. The position of the focus point region is very sensitive to the value of the top quark mass that enters the RGE and also differs for different RGE codes [16, 31, 32]. For the case of SOFTSUSY used here, rather low values of the top quark mass are necessary to reach the region where $\mu$ drops rapidly, at least for intermediate values of $\tan \beta$. As displayed in Fig. 2, for $m_{t}=175 \mathrm{GeV}$, one finds a cosmologically allowed region at large $M_{0}$ only for large $\tan \beta$. In the focus point region, the main annihilation channels are $\tilde{\chi}_{1}^{0} \tilde{\chi}_{1}^{0} \rightarrow W^{+} W^{-}, Z Z$. Coannihilation channels such as $\tilde{\chi}_{1}^{0} \tilde{\chi}_{1}^{ \pm} \rightarrow q q^{\prime}$ can also help reduce the relic density. One also finds an important contribution from $\tilde{\chi}_{1}^{0} \tilde{\chi}_{1}^{0} \rightarrow t \bar{t}$ as soon as this channel becomes kinematically accessible. This occurs, in the focus point region, when $M_{0} \approx 4.5-5 \mathrm{TeV}$, Fig. 2 $\mathrm{d}$. Even though the stops are heavy $\left(m_{\tilde{t}_{1}} \approx 2.5 \mathrm{TeV}\right)$, a sufficiently large annihilation cross section results from the $\mathrm{Z}$ exchange contribution. For the gauge bosons annihilation channels, values consistent with WMAP for $\Omega h^{2}$ require not so large a value for $\mu$. This implies that the chargino and lightest neutralinos should be rather light. For example, the chargino and neutralino are confined to have $m_{\tilde{\chi}_{1}^{+}}<375 \mathrm{GeV}$ for $\tan \beta=50, m_{t}=175 \mathrm{GeV}$. The chargino and neutralino can be nearly degenerate $\mathcal{O}(10) \mathrm{GeV}$ but this occurs mainly when the relic density falls below the WMAP range. For $\Omega h^{2} \approx .1$ typical mass differences are rather around $50 \mathrm{GeV}$. Note that models in the focus point region feature a very heavy sfermion sector, the LHC will have little opportunity 


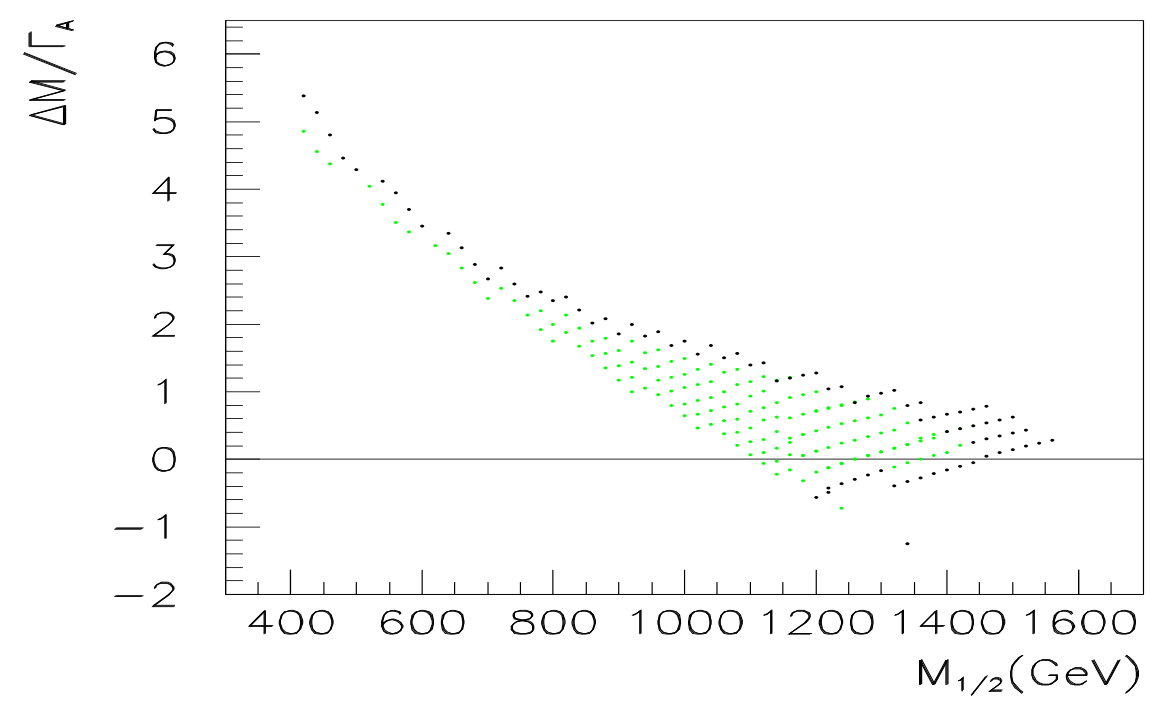

Figure 4: Mass difference relative to the pseudoscalar width $\left(\Delta M_{A \tilde{\chi}_{1}^{0}} / \Gamma_{A}\right)$ as function of $M_{1 / 2}$ for $\tan \beta=50, \mu>0, A_{0}=0$. Constraints from LEP as well as $b \rightarrow$ s $\gamma$ are taken into account. Models within (below) the WMAP range are labelled by black (green/grey) dots.

to discover the squarks, only the gluino could be accessible. At the same time the rather light Higgsino sector could be probed at a linear collider, extending the reach of the LHC 84. Furthermore these models are also interesting for future direct dark matter detection experiments as will be discussed in Section 5 .

\subsection{2 $m_{t} \neq 175 \mathrm{GeV}$}

The position of the allowed region at large $M_{0}$ is the main qualitative difference between mSUGRA models with a lower input values for the top quark mass while the more stringent constraint originating from the light Higgs mass shifts the range of allowed values for sparticle masses in the low $M_{0}$ region. For example, when $m_{t}=170 \mathrm{GeV}$, one finds a focus point region even at $\tan \beta=10$, Fig. 5 . At large values of $\tan \beta$, the focus point region becomes much wider. Indeed all points between the contour delimiting the region forbidden by the requirement of electroweak symmetry breaking (EWSB) and $\Omega h^{2}=.094$ have a value for the relic density below the WMAP range, indicating eventually some other dark matter component. The shape of the allowed region at $\tan \beta=50$ differs significantly from the case with heavier top quark mass. First, one tends to get a lighter heavy Higgs, extending the Higgs funnel region towards higher values of $M_{0}$, at the same time shifts in $\mu$ are found pushing the focus point towards lower values of $M_{0}$. This is solely an effect of the RGE dependence of the pseudoscalar mass and of $\mu$ on the top quark mass. As concerns the relic density constraint the requirement remains the same, only models where the neutralino lies near $M_{A} / 2$, roughly $\Delta M / \Gamma_{A} \approx 2$ are allowed. The Higgs funnel and focus point regions merge for $M_{1 / 2} \approx 1 \mathrm{TeV}$. Furthermore, because of the additional contribution from Higgs exchange, one finds acceptable values for the relic density in the low $M_{0}-M_{1 / 2}$ region. In the end, a large fraction of parameter space is allowed by the relic density constraint. Of course direct measurements such as $b \rightarrow s \gamma$ play an important role here particularly in the low $M_{0}-M_{1 / 2}$ region. 

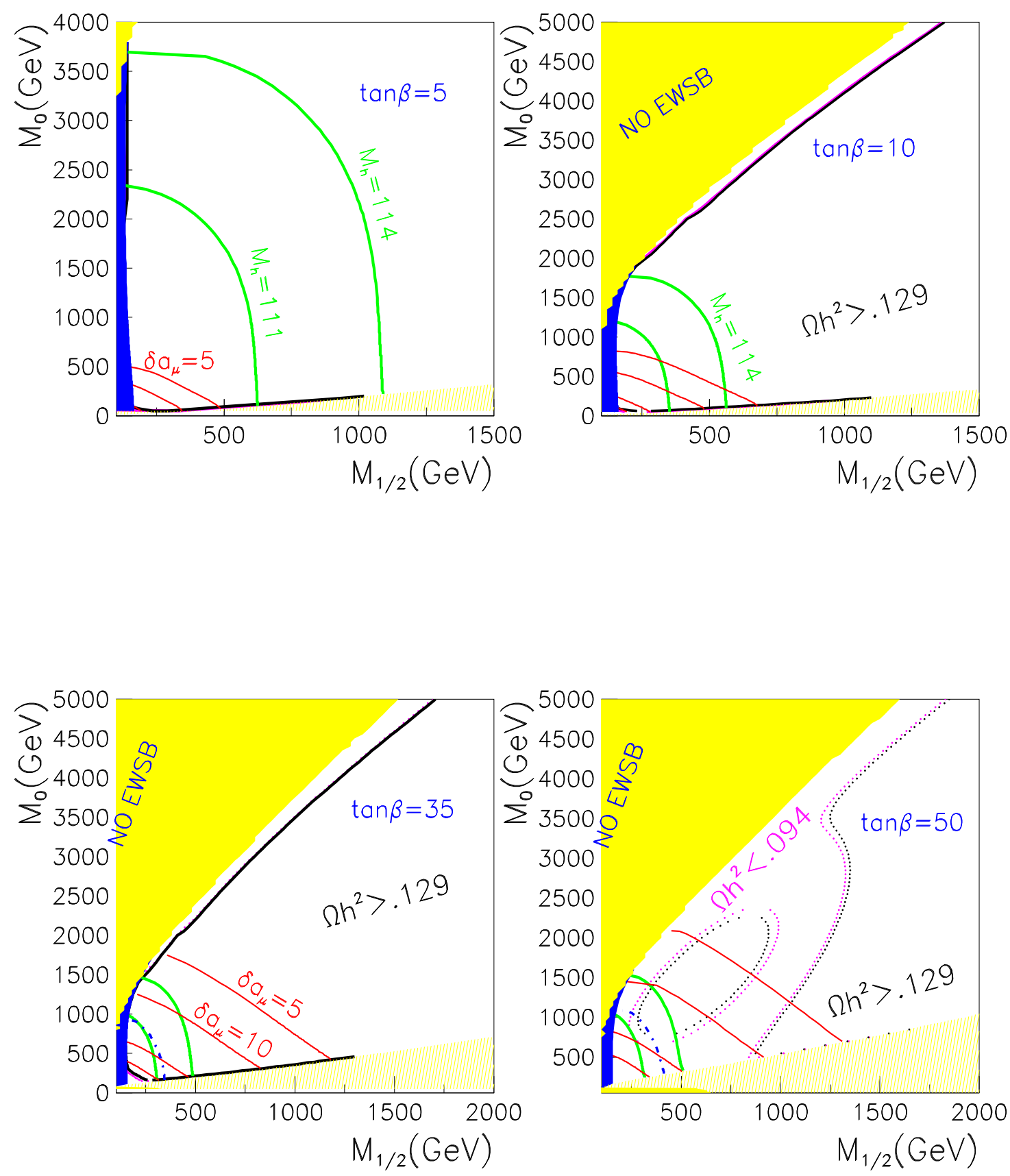

Figure 5: Same as in Fig. 2 for $m_{t}=170 \mathrm{GeV}$ 
Conversely a heavier top quark, say $m_{t}=179 \mathrm{GeV}$, means a shift of the focus point region towards higher values of $M_{0}$ [85]. We have stopped our scans at $M_{0}=5 \mathrm{TeV}$, as we found it rather difficult to obtain a solution to the RGE that converged rapidly for very large $M_{0}{ }^{5}$. The relaxed bound from the Higgs mass also favours the region of parameter space where not so heavy neutralinos/charginos and sleptons can be found, here $m_{\tilde{\chi}_{1}^{0}}>90 \mathrm{GeV}$ for $\tan \beta=10$. Finally the Higgs funnel moves closer to the coannihilation region, indeed the heavy Higgs masses are shifted upwards with the increase of the top Yukawa making it increasingly difficult to have $m_{\tilde{\chi}_{1}^{0}} \approx M_{A} / 2$. Then for $\tan \beta=50$ the allowed region only extends slightly above the stau coannihilation region.

mSUGRA, the case $A_{0} \neq 0$

For simplicity we have up to now fixed the value of the trilinear coupling to $A_{0}=0$. Although the constraints are not extremely sensitive to this parameter yet as one varies $A_{0}$ over the full range $\left|A_{0}\right|<2 \mathrm{TeV}$, one sees an impact on the allowed regions in the $M_{0}-M_{1 / 2}$ plane. For one, a non-zero value for the trilinear coupling shifts the physically allowed region where the neutralino is the LSP. First, a region with tachyons appears at low $M_{1 / 2}$. Furthermore, the stau mass is lowered since the trilinear mixing affects the running of the $\tilde{\tau}_{L, R}$ masses as well as the running of $\mu$ which determines the mixing in the stau sector. Indeed the mixing is proportionnal to $A_{\tau}+\mu \tan \beta$ so is usually dominated by the term $\mu \tan \beta$. The boundary of the coannihilation region then moves towards higher values of $M_{0}$ for non-zero values of $A_{0}$. This is particularly obvious at large tan $\beta$, Fig. 7, 8 ,

First consider models with $A_{0}=2000 \mathrm{GeV}$ and $m_{t}=175 \mathrm{GeV}$. One finds that consistency with WMAP is limited to a region just at the boundary of the theoretically excluded region for $\tan \beta=10$, see Fig. 7 . One finds a new feature in these scenarios, a region where the $\tilde{t}_{1}$ is the NLSP. This region which occurs at moderate values of $M_{0}$ lies just to the right of the region where one finds tachyonic solutions, i.e. for $200<M_{0}<$ $650 \mathrm{GeV}$. The main annihilation for neutralinos is into fermion pairs and coannihilation channels such as $\tilde{\chi}_{1}^{0} \tilde{t}_{1} \rightarrow t g, W^{+} \bar{b}$ contribute also significantly. Note that the contribution from $\tilde{\chi}_{1}^{0} \tilde{t}_{1} \rightarrow W^{+} \bar{b}$ can give the dominant contribution to the thermally averaged cross section exceeding the QCD channels with gluon emission ${ }^{6}$. Then one can satisfy the relic density constraint because of the coannihilation processes involving stop quarks. However this stop coannihilation region is not allowed by the light Higgs mass constraint. This constraint is more severe than in models where the trilinear coupling vanishes, only the stau coannihilation region remains once the $m_{h}$ limit is imposed. The allowed values for neutralinos masses in the coannihilation region lie in the range $170(126)<m_{\tilde{\chi}_{1}^{0}}<$ $440(700) \mathrm{GeV}$ for $\tan \beta=10(50)$. Of course one can have models with an even heavier LSP since for $\tan \beta=50$, both the focus point region as well as the Higgs funnel are present. These regions are shifted as compared to the $A_{0}=0$ case, in particular the heavy Higgs masses are shifted down making for a more efficient annihilation of neutralinos via a heavy Higgs exchange. Then neutralinos as heavy as $m_{\tilde{\chi}_{1}^{0}} \approx 740 \mathrm{GeV}$ are allowed.

For negative values of $A_{0}$, say $A_{0}=-1 \mathrm{TeV}$, the constraints from the Higgs mass are relaxed while the $b \rightarrow s \gamma$ is more important as was discussed in the previous section. The relic density is the main constraint on models with low $\tan \beta$. Again one finds a region where the $\tilde{t}_{1}$ is the LSP at low $M_{0}-M_{1 / 2}$ values. Just when the $\tilde{t}_{1}$ becomes the NLSP,

\footnotetext{
${ }^{5}$ The predictions of the different codes for the SUSY spectrum calculation can be very different in this region [31. For example, with ISAJET the authors of [85] find the focus point region all the way up to $M_{0}=10 \mathrm{TeV}$.

${ }^{6}$ This contribution is not included in $[86]$.
} 

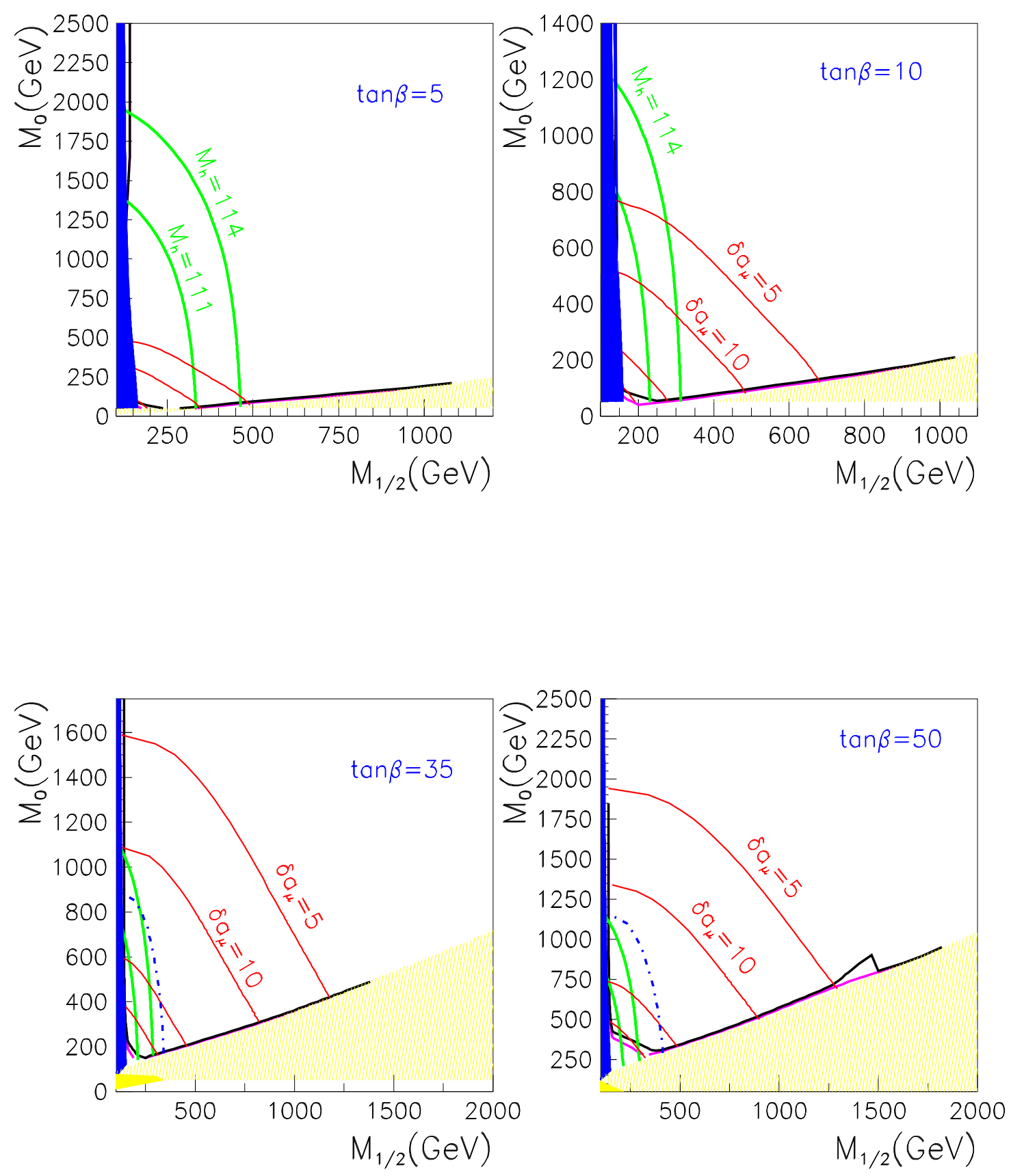

Figure 6: Same as in Fig. 2 for $m_{t}=179 \mathrm{GeV}$. 

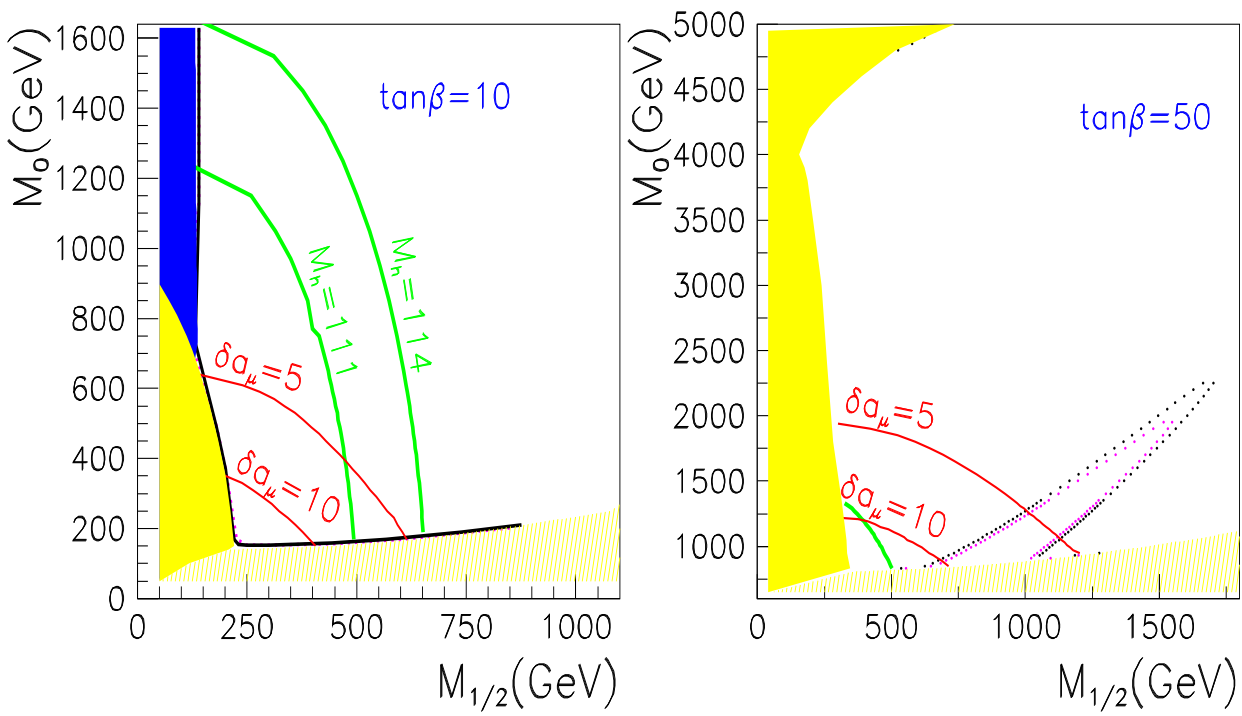

Figure 7: Same as in Fig. Q for $\mu>0, m_{t}=175 \mathrm{GeV}$ and $A_{0}=2000 \mathrm{GeV}$. Only the contours $\delta a_{\mu}=5,10 \times 10^{-10}$ (top to bottom) can be seen.

values of the relic density consistent with WMAP are found, see Fig 8 for $\tan \beta=10$. However this region is barely compatible with the Higgs mass limit and does not pass the $b \rightarrow s \gamma$ constraint. One also finds as usual a thin $\tilde{\tau}_{1}$ coannihilation region. Furthermore, with the large negative mixing which tends to increase the heavy Higgs masses, the Higgs funnel is reduced to a tiny region even at $\tan \beta=50$, Fig. 8 . Note also that the constraint from $B_{s} \rightarrow \mu^{+} \mu^{-}$starts to restrict the mSUGRA parameter space although it is not yet competitive with other direct constraints. For larger values of the mixing parameter, e.g. $A_{0}=-1700 \mathrm{GeV}$, it is possible to find models that satisfy all constraints and where the $\tilde{t}_{1}$ is the NLSP [87.

\section{$3.3 \quad \chi^{2}$ fit}

So far we have discussed the effect of each type of constraint taken individually as well as their sensitivity on the top quark mass. To get a better estimate of the impact of the uncertainty on the input parameters, we have also performed a $\chi^{2}$ fit to the four observables: $\Omega h^{2}, b \rightarrow s \gamma, m_{h}$ and $m_{t}$ for a fixed value of $\tan \beta$ and $A_{0}$. We use $m_{t}=$ $174.3 \pm 5.1 \mathrm{GeV}$. We show in Fig. 9 the allowed area at $68 \%$ and $95 \%$ C.L. One finds that the favoured regions are the coannihilation as well as the focus point region for $\tan \beta=10$. The area of parameter space allowed at $95 \%$ C.L. after allowing for the $m_{t}$ uncertainty now seems very wide especially at $M_{0}>2 \mathrm{TeV}$. This reflects the high sensitivity of the RGE to the value of the top quark mass. For $\tan \beta=50$, the $\tilde{\tau}_{1}$-coannihilation, Higgs funnel and focus point region are not easily distinguishable and a large fraction of parameter space is allowed. Note that both the Higgs funnel and focus point regions are very sensitive to the value of the top quark mass as we have seen in Fig. 256. 

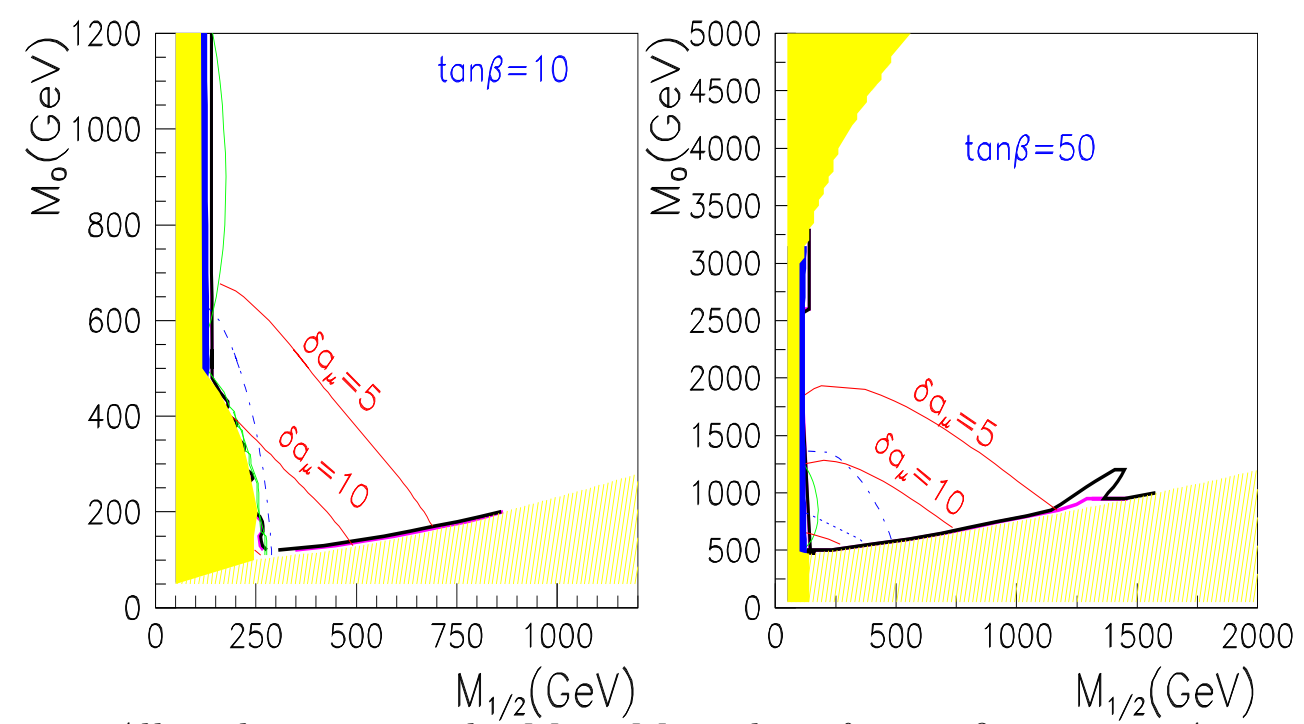

Figure 8: Allowed regions in the $M_{0}-M_{1 / 2}$ plane for $\tan \beta=10,50, A_{0}=-1000 \mathrm{GeV}$, $\mu>0$ and $m_{t}=175 \mathrm{GeV}$. Contours for $\Omega h^{2}=.129(.094)($ black $/$ pink dots $) m_{h}=114 \mathrm{GeV}$ (green) $\delta a_{\mu}=5,10,30 \times 10^{-10}$ (red) (top to bottom), $b \rightarrow s \gamma=2.25 \times 10^{-4}$ (dash-dot/blue) and $B_{s} \rightarrow \mu^{+} \mu^{-}=2 \times 10^{-7}$ (dots) for $\tan \beta=50$. The exclusion regions are labelled in the same manner as in Fig. 國.
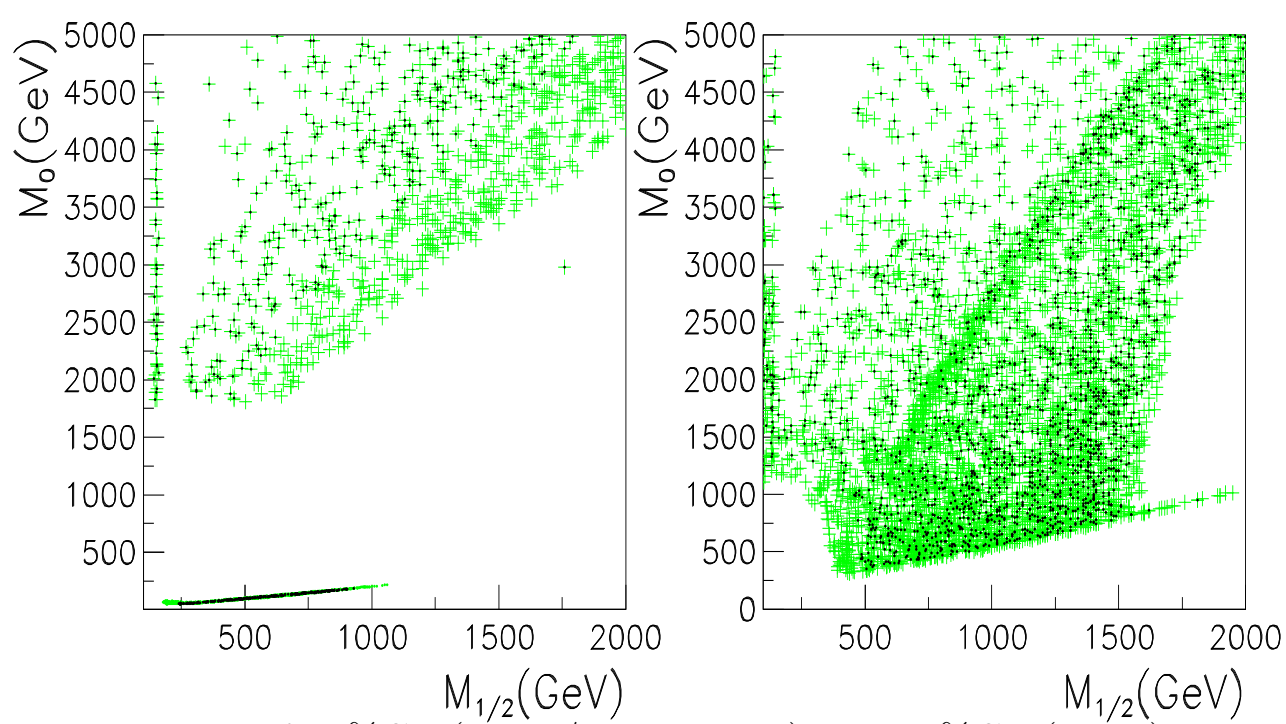

Figure 9: Regions of 95\%CL (green/grey crosses) and $68 \% \mathrm{CL}$ (black) in the $M_{0}-M_{1 / 2}$ plane from a $\chi^{2}$ fit to $\Omega h^{2}, m_{h}, \operatorname{Br}(b \rightarrow s \gamma)$ and $m_{t}$ for $\tan \beta=10$ (left) and $\tan \beta=50$ (right), here $\mu>0$ and $A_{0}=0$. 
In summary, in mSUGRA models a combination of the direct collider limits and relic density constraints, restricts the allowed models in very specific regions of parameter space. The exact location of these regions in the $M_{0}-M_{1 / 2}$ plane is however sensitive to theoretical uncertainties in the evaluation of the spectrum. Nevertheless the predictions at the EW scale are rather contrived: either nearly degenerate $\tilde{\tau} /$ neutralinos, light higgsino-type neutralinos or at large $\tan \beta$, neutralinos that are near $M_{A} / 2$. The results we have presented agree qualitatively with other published results, however differences in the position of the heavy Higgs funnel as well as the focus point region are found [4, 88. To a large extent these differences can be traced back to differences in the evaluation of the supersymmetric spectrum at the EW scale. These issues were brought up in Ref. 31] and more details will appear in 32 .

\section{Non-universal gauginos}

In models where one relaxes the gaugino universality conditions the allowed regions in parameter space can change drastically [89, 90, 91]. For one, the wino content of the LSP can be significantly increased, favouring the annihilation cross section into pairs of gauge bosons. This occurs for example for $M_{1}^{\prime} / M_{2}^{\prime}>1$ at the GUT scale. Then reasonable values for the relic density can be obtained even for heavy sleptons. The annihilation cross section into pairs of $\mathrm{W}$ tends to be more efficient than in lepton pairs 92 in fact so efficient that the relic density often falls below the WMAP range. Furthermore, the coannihilation with heavier charginos and neutralinos, also the most efficient ones, are much more frequent in this type of model. Second, as in the universal case, one finds significant areas of parameter space where neutralino annihilation proceeds through a schannel Higgs exchange. However these regions do not necessarily only appear at large $\tan \beta$. Allowing $M_{3}^{\prime} / M_{2}^{\prime}<1$ at the GUT scale reduces the value of the heavy Higgs masses without much effect on the neutralino mass. This type of models also predicts smaller values for $\mu$ which means a LSP with a significant Higgsino component and more efficient annihilation channels without requiring high $\tan \beta$ [93. Alternatively, reducing $M_{1}^{\prime} / M_{2}^{\prime}$ allows annihilation of neutralino through a light Higgs exchange. Finally nonuniversality in the gaugino sector can also affect the predictions for the sfermion masses. In particular reducing the parameter $M_{3}^{\prime}$ for given $M_{1}^{\prime}, M_{2}^{\prime}$ values, produces much lighter squarks. Coannihilation channels with squarks then are more likely especially when there is a large mixing in the squark sector. Altogether one finds the same mechanisms as in mSUGRA for getting sufficient annihilation/coannihilation of neutralinos but the allowed region in the parameter space of the MSSM shifts significantly and no longer requires a fine tuning of parameters. The direct constraints are of course also modified in non-universal models. This will be detailed next and illustrated with a few typical case studies. Here we only consider the case $m_{t}=175 \mathrm{GeV}$.

\section{1 $\quad M_{3}^{\prime}>M_{2}^{\prime}$, the case $M_{3}^{\prime}=2 M_{2}^{\prime}=2 M_{1}^{\prime}$}

By changing only $M_{3}^{\prime}$ while keeping $M_{2}^{\prime}=M_{1}^{\prime}$ at the GUT scale means that at the weak scale one retains the usual relation $M_{1} \approx 0.4 M_{2}$. Futhermore the parameter $\mu$ tends to be larger than in mSUGRA, see Eq. 5. Therefore the $\tilde{\chi}_{1}^{0}$ is nearly a pure bino. The relic density constraint then becomes more severe than in the universal case since annihilation 
of bino LSP's into light fermions, although dominant, is not sufficient to bring $\Omega h^{2}$ in the WMAP range. The pseudoscalar mass, which is driven by $M_{3}$, is also much heavier than in the universal case so annihilation through the heavy Higgs cannot take place. The focus point region is pushed towards higher values of $M_{0}$ even at $\tan \beta=50$. Then the only allowed region is the $\tilde{\tau}_{1} / \tilde{\chi}_{1}^{0}$ coannihilation region. This region is always present since the relation between $M_{0}$ and $M_{1}, M_{2}$ which to a large extent determines $m_{\tilde{\chi}_{1}^{0}}, m_{\tilde{\tau}_{1}}$ has not changed. The allowed narrow band extends beyond $M_{1 / 2}=2 \mathrm{TeV}$, see Fig [10, so that a rather heavy SUSY spectrum is possible. In the coannihilation region one finds roughly $\Delta M_{\tilde{\tau} \tilde{\chi}_{1}^{0}} \approx 5-10 \mathrm{GeV}$ for $\tan \beta=10$, comparable to the values found for the universal case. For larger values of $\tan \beta$, the increased contribution of many channels, in particular those with a light Higgs, $\tilde{\chi}_{1}^{0} \tilde{\tau} \rightarrow \tau h, \tilde{\tau} \tilde{\tau} \rightarrow h h$ means that larger mass differences can be compatible with WMAP.

On the other hand the constraints from colliders are not as severe. As the Higgs mass is driven by the squark mass which increases with $M_{3}^{\prime}$, lower values of $M_{1 / 2}=M_{2}^{\prime}$ should be allowed. The upper bound on the Higgs mass is indeed easily satisfied for $\tan \beta=10$ as can be seen in Fig[10. Allowed regions are found even at $\tan \beta=5$. There for $m_{h}>111 \mathrm{GeV}$ one recovers a small allowed region with $M_{1 / 2}>250 \mathrm{GeV}$ and light sleptons. The constraints from precision measurements are easily satisfied in this model since heavy squarks also imply that $b \rightarrow s \gamma$ is close to the standard model prediction. Only a tiny region is excluded for $\tan \beta=50$. In this type of model, for example when $M_{1 / 2}>800 \mathrm{GeV}$, the heavy squarks make it difficult for the LHC to discover supersymmetry. On the other hand one recovers part of the low $M_{1 / 2}$ region that is favourable for collider searches of charginos and sfermions.
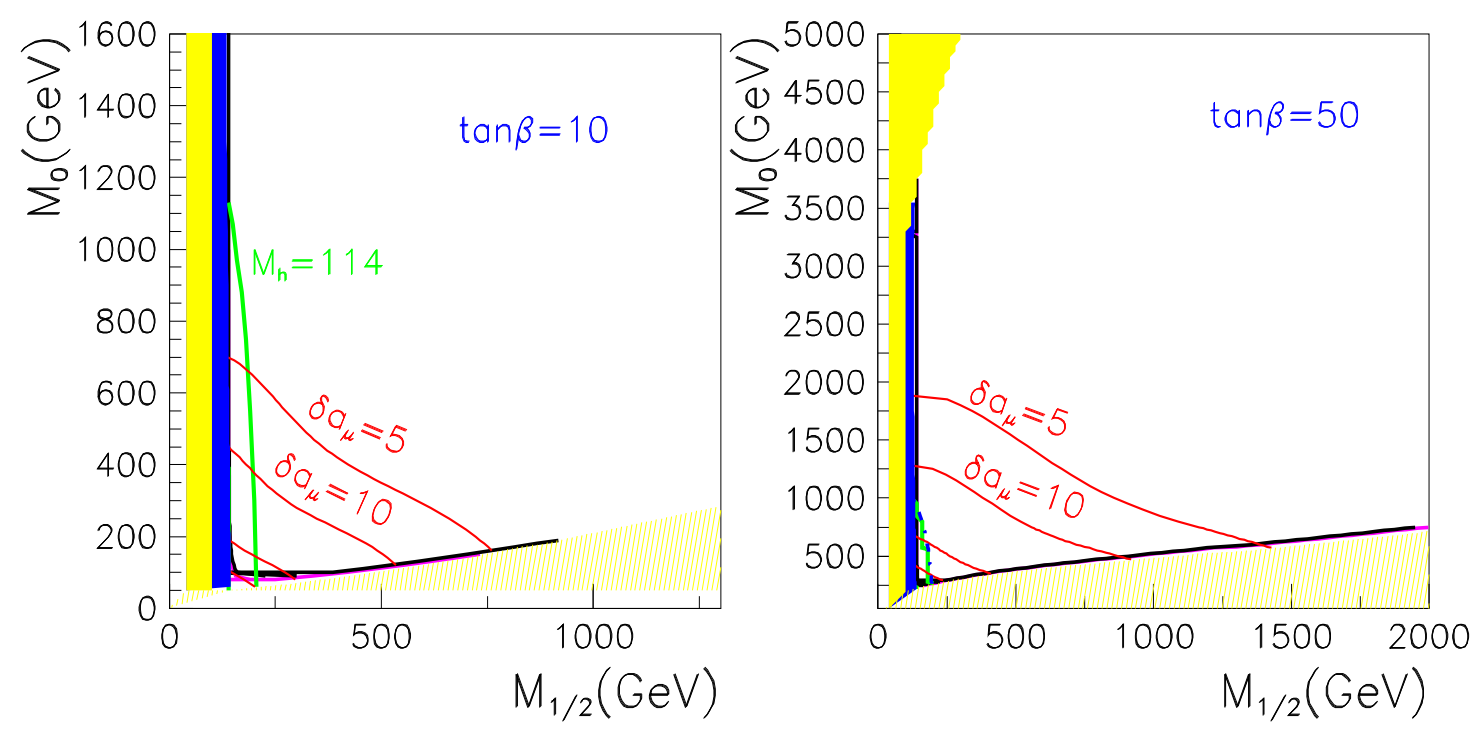

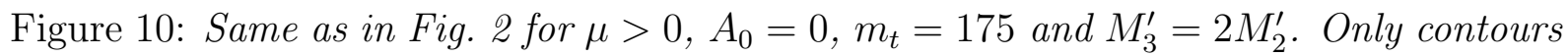
for $m_{h}=114 \mathrm{GeV}$ (green) are relevant.

\section{2 $M_{3}^{\prime}<M_{2}^{\prime}$, the case $M_{3}^{\prime}=M_{2}^{\prime} / 2$}

Decreasing the value of $M_{3}^{\prime}$ while keeping $M_{2}^{\prime}=M_{1}^{\prime}$ at the GUT scale means that the relic density constraint can be satisfied more easily. First, one finds a neutralino-LSP 
with higher Higgsino component, although the LSP remains mainly a bino. Indeed $\mu$ is typically smaller than in unified models, Eq 5 . More importantly one feels the presence of the heavy Higgs pole even for moderate values of $\tan \beta$. As we have discussed in Section 1. solutions for $M_{A} \approx 2 m_{\tilde{\chi}_{1}^{0}}$ can be found even for $\tan \beta=10$, Eq. 6. Neutralino annihilation into fermions is then dominated by the s-channel scalar exchange and the main channels are $\tilde{\chi}_{1}^{0} \tilde{\chi}_{1}^{0} \rightarrow t \bar{t}, b \bar{b}, \tau \bar{\tau}$, the former being dominant at lower values of $\tan \beta$ only. Note that for $\tan \beta=10$ the coupling of the pseudoscalar to $b$ quarks is already 2 to 3 times larger than to $\mathrm{t}$ quarks the ratio $\frac{A b b}{A t t} \approx \frac{m_{b}}{m_{t}} \tan \beta^{2}$ and this mode starts to dominate. The region that in the universal case was called the $\tilde{\tau}$ coannihilation region now becomes a Higgs/coannihilation one and includes the low $M_{0}-M_{1 / 2}$ region, similar to what was observed previously for large values of $\tan \beta$. In fact as one moves closer to the $\tilde{\tau}$ LSP region and coannihilation channels start to contribute significantly to the effective cross section, the relic density is already much below the WMAP range. This corresponds in Fig 11] to the region between the $\tilde{\tau}$ LSP line and the contour $\Omega h^{2}=.094$. This means that values of $\Omega h^{2}$ within the range of WMAP are no longer associated with nearly degenerate $\tilde{\tau}-\tilde{\chi}_{1}^{0}$. As in mSUGRA, as one moves towards larger values of $M_{1 / 2}$ the stau coannihilation cross sections become much smaller and even models with completely degenerate stau/neutralino cannot meet the upper limit of WMAP on the relic density. However a new Higgs funnel region appears, it corresponds again to s-channel Higgs resonance but this time for the coannihilation processes $\tilde{\chi}_{1}^{0} \tilde{\chi}_{2}^{0}, \tilde{\chi}_{1}^{0} \tilde{\chi}_{3}^{0} \rightarrow t \bar{t}, b \bar{b}, \tau \bar{\tau}$ as well as charged Higgs exchange in $\tilde{\chi}_{1}^{0} \tilde{\chi}_{1}^{+} \rightarrow t \bar{b}$. The onset of the charged Higgs exchange contribution is easily visible as a kink in the contours of constant relic density in Fig. [11-b. The focus point region is also more important even for $\tan \beta=10$.

As one increases $\tan \beta$, the heavy Higgs decay into b-quarks completely dominates. The allowed region in the $M_{0}-M_{1 / 2}$ plane correspond mainly to annihilation into $b \bar{b}, \tau \bar{\tau}$. One does not find a region where $\tilde{\tau}$-coannihilation dominates, although $\tilde{\tau}-\tilde{\chi}_{1}^{0}$ can be quite close in mass, this occurs only when one is near the Higgs resonance. Then the relic density is much below the WMAP range. For values of $M_{1 / 2}>1100 \mathrm{GeV}$, only annihilation or coannihilation with heavier neutralinos/charginos into fermion pairs through a Higgs exchange take place. Since the value of $\mu$ is smaller than in the universal case, there is also a more important contribution from the annihilation into gauge bosons channel and the focus point region is much wider for a fixed top quark mass. Eventually the focus point and Higgs annihilation region merge, for example for $\tan \beta=35$ and for $M_{1 / 2} \approx 2 \mathrm{TeV}$ the entire range of $M_{0}$ is below the WMAP upper bound. For $\tan \beta=50$, the merging of the two regions is complete and a large fraction of the parameter space is allowed. As for other values of $\tan \beta$, annihilation into $b \bar{b}$ pairs dominates for low values of $M_{0}$ and annihilation (or coannihilation) into gauge boson pairs eventually takes over as one moves close to the region where EWSB cannot take place. We remark that even with a moderate trilinear mixing, say $A_{0}=-600 \mathrm{GeV}$, one finds regions where the $\tilde{t_{1}}$ is the NLSP.

As one would expect, when $M_{3}^{\prime}<M_{2}^{\prime}$, the Higgs mass constraint becomes more severe as the squark masses are lower than in the universal case for a fixed value of $M_{1 / 2}=$ $M_{2}^{\prime}$. For example, for $\tan \beta=10$, only $M_{1 / 2}>460 \mathrm{GeV}$ is allowed in the coannihilation region. The lighter squarks relative to the universal case imply a larger deviation from the standard model prediction for $b \rightarrow s \gamma$ as well. The exclusion region in the left-hand corner of the $M_{0}-M_{1 / 2}$ plane where the $b \rightarrow s \gamma$ branching ratio drops below the allowed range becomes more important. As usual this constraint takes over the one coming from the Higgs mass as one increases $\tan \beta$. For $\delta a_{\mu}$, the main difference from the universal case is that the value of $\mu$ are modified by the RGE's. Nevertheless the allowed region 
is very similar to the one obtained in the universal case, the contours are only slightly shifted towards higher $M_{1 / 2}$ values. Note that here again requiring a strict deviation from the standard model, would rule out most of the region at large $M_{0}-M_{1 / 2}$. Combining this with other direct constraints would leave only a small region of parameter space for $\tan \beta \leq 10$ as the direct constraints are more effective in this class of model.

In these models typically squarks are lighter than in mSUGRA, one then expects a good discovery potential for the LHC. However as one easily finds a region where $m_{\tilde{\chi}_{1}^{0}} \approx M_{A} / 2$, even for very heavy neutralinos one can find reasonable values for the relic density. It is possible then to obtain a very heavy supersymmetric spectrum. For example for $M_{0}=4 \mathrm{TeV}, M_{1 / 2}=3 \mathrm{TeV}$, the spectrum consists of heavy squarks, $m_{\tilde{q}}=3-4 \mathrm{TeV}$, neutralinos and charginos, $m_{\tilde{\chi}_{1}^{0}} \approx m_{\tilde{\chi}_{1}^{+}} \approx 820 \mathrm{GeV}$ and also heavy Higgses $m_{H} \approx m_{H^{+}} \approx$ $m_{A} \approx 1.5 \mathrm{TeV}$, a difficult task for discovering supersymmetry at colliders. The only new particle that could be reached at a collider would be the light Higgs. In these models even $\delta a_{\mu}$ does not differ significantly from zero. Only an unambiguous evidence for a non-zero contribution to $\delta a_{\mu}$ would restrict this class of models with heavy sparticles.

\subsection{The general case $M_{3}^{\prime} \neq M_{2}^{\prime}=M_{1}^{\prime}$}

There is a smooth transition from the region with universality where $\Omega h^{2}$ is in general too large to the region $M_{3}^{\prime}<M_{2}^{\prime}$ where there is not enough relic density. This is illustrated in Fig. 12 showing the value of $\Omega h^{2}$ as function of $r_{32}$ for three typical values of $M_{0}=$ $300,1500,3000 \mathrm{GeV}$. For these values of $M_{0}$ one crosses the coannihilation, Higgs exchange and focus point region respectively for $m_{t}=175 \mathrm{GeV}$. Clearly it is much easier to satisfy the relic density upper bound in models where $M_{3}^{\prime}<M_{2}^{\prime}$. As just discussed this is the effect of the heavy Higgs pole and of the more important Higgsino component of the bino LSP. The Higgs funnel can be found even for $M_{0}=300 \mathrm{GeV}$ as long as $r_{32}<.5$. Furthermore for $\tan \beta=50$, and $M_{0}=3000 \mathrm{GeV}$, nearly all models are below the WMAP range when $r_{32}<.4$, this is a combined effect of a smaller value for $\mu$ and $M_{A}$ as compared to the universal case.

In conclusion, relaxing the unification condition on $M_{3}^{\prime} / M_{2}^{\prime}$ while keeping $M_{1}^{\prime}=M_{2}^{\prime}$ helps recover some values for low $\tan \beta$ because of either a weaker Higgs mass limit or possible annihilation through a heavy Higgs resonance. This was illustrated for $\tan \beta=5$. Furthermore, the case $M_{3}^{\prime}<M_{2}^{\prime}$ satisfies more easily the relic density constraints for any values of $\tan \beta$. In particular for large $\tan \beta$ where the relic density constraint is satisfied in a large portion of the available parameter space. A significant fraction of the $r_{32}<1$ models then predict squarks within the range accessible by the LHC. On the other hand because of annihilation/coannihilation through the heavy Higgses, scenarios with heavy neutralinos as well as heavy sfermions can satisfy all constraints while predicting few signals at colliders. In these models a value of $\delta a_{\mu}$ compatible with the standard model is predicted, evidence of a deviation might be the best way to rule them out.

\subsection{The case $M_{1}^{\prime} \neq M_{2}^{\prime}=M_{3}^{\prime}$}

We now restore the unification condition $M_{3}^{\prime}=M_{2}^{\prime}$ while allowing $M_{1}^{\prime}$ to differ from $M_{2}^{\prime}$ at the GUT scale. This will directly affect the wino/bino nature of the LSP. If the LSP has a higher wino component, which occurs in models where $M_{1} \geq M_{2}$ at the weak 

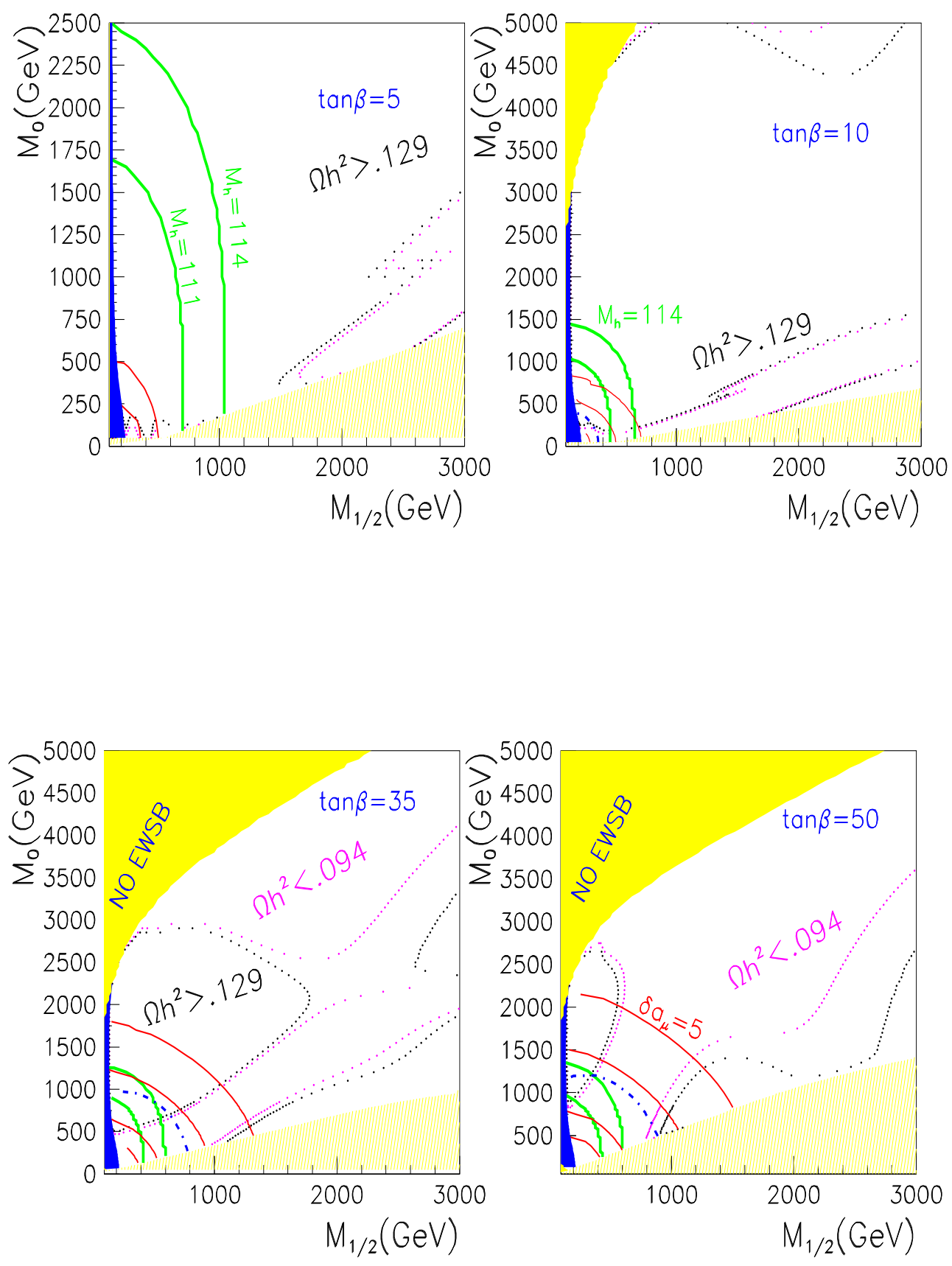

Figure 11: Same as Figure 2 for $\mu>0, A_{0}=0, m_{t}=175$ GeV and $M_{3}^{\prime}=M_{2}^{\prime} / 2$. 

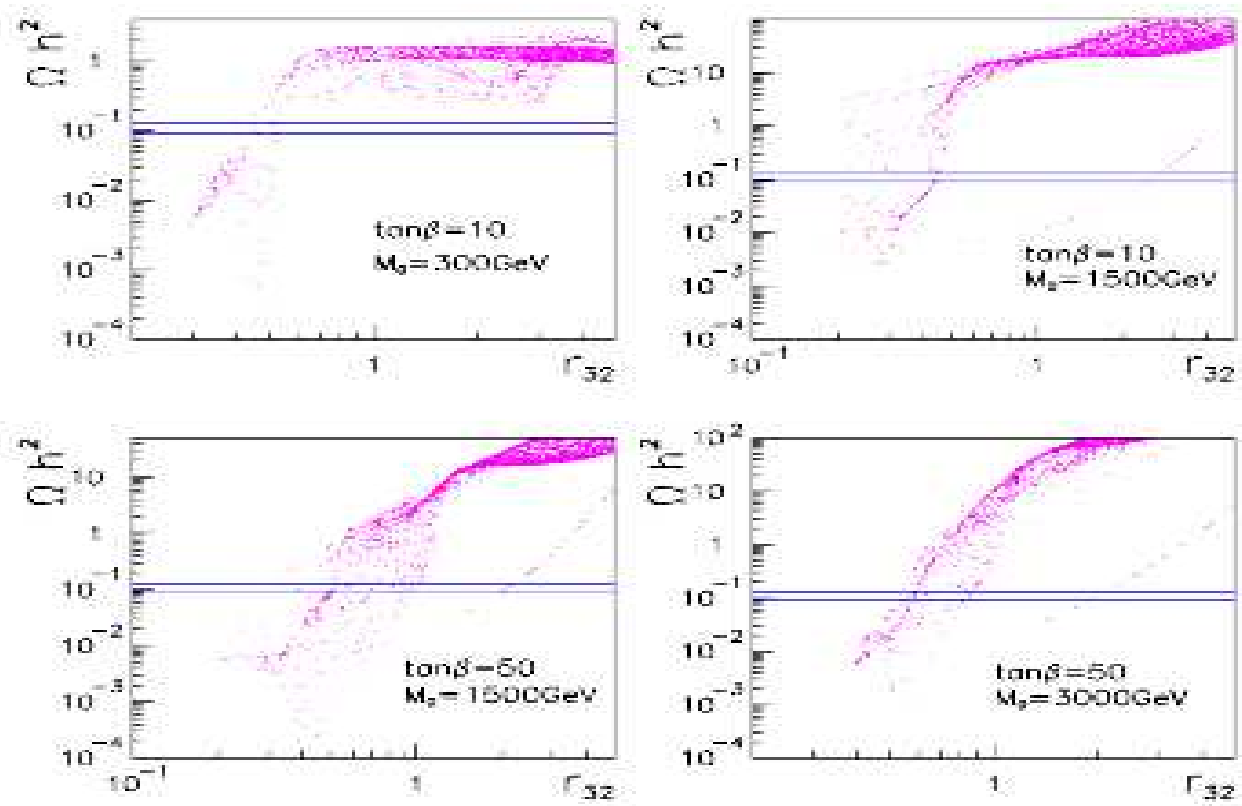

Figure 12: $\Omega h^{2}$ vs $r_{32}$ for $\tan \beta=10, M_{0}=300,1500 \mathrm{GeV}$ and $\tan \beta=50, M_{0}=$ $1500,3000 \mathrm{GeV}$. In all cases, $\mu>0, A_{0}=0, m t=175 \mathrm{GeV}$ and the constraints from $b \rightarrow s \gamma, m_{h}$ as well as LEP direct limits are imposed.

scale, annihilation into $\mathrm{W}$ pairs becomes important especially since LEP constraints forces $m_{\tilde{\chi}_{1}^{0}}>M_{W}$. Furthermore this process is much more efficient than the annihilation into fermion pairs that dominated for a bino LSP. A wino LSP also opens up the coannihilation channels with other gauginos. Then one expects the relic density constraint to be easily satisfied in models where $M_{1}^{\prime}>M_{2}^{\prime}$. For fixed values of $M_{0}$, we scanned over models with $.2<r_{12}<5$ and $\tan \beta=10,50$. Fig 13 clearly shows the dramatic effect on $\Omega h^{2}$ of increasing $r_{12}$, varying from almost always too large relic density when $r_{12}<1$ to nearly always too small when $r_{12}>>1$ passing by an intermediate region where $\Omega h^{2}$ lies within the desired range. This value preferred by WMAP is around $r_{12}=1.8$ for $\tan \beta=10$ and corresponds to $M_{1} \approx M_{2}$ at the electroweak scale. Thus the lightest neutralino is a mixed bino/wino. We will study this case in more details below. Note that we have stopped our scans at $M_{2}=2 \mathrm{TeV}$ so that we have not allowed very heavy neutralinos. Had we extended the parameter space it would have been possible to find models with very heavy neutralinos that fell within the range of WMAP even for $r_{12}>2$. For higher values of $\tan \beta$ roughly the same behaviour is observed, only then a wider selection of models passes the constraint. For example, for $\tan \beta=50$ and $M_{0}=1500 \mathrm{GeV}$, many models satisfy the relic density constraint, this reflects the presence of the Higgs funnel which appears already for $r_{12} \approx 1$. 

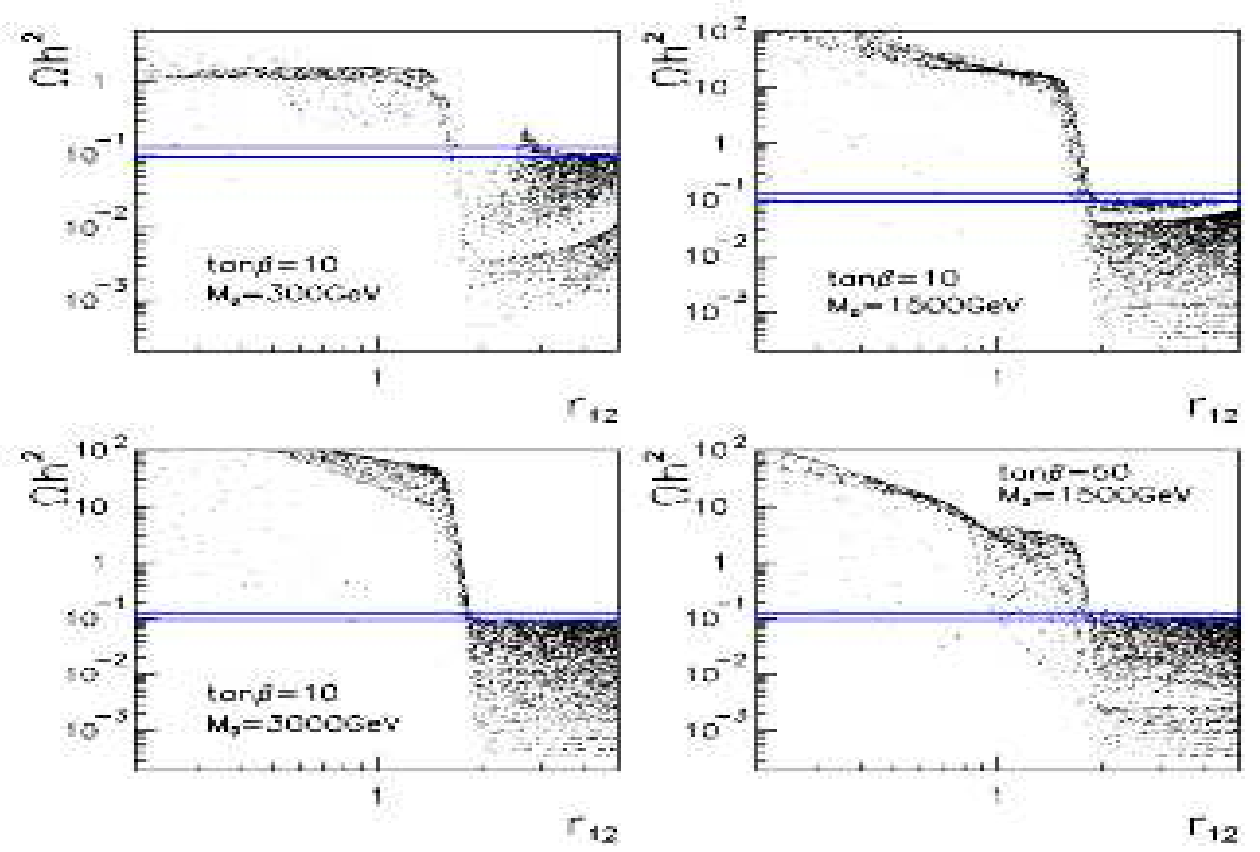

Figure 13: Relic density vs $r_{12}$ in models with $\mu>0, A_{0}=0, m t=175$ GeV for $a-c$ ) $\tan \beta=10$ and $\left.M_{0}=300,1500,3000 \mathrm{GeV} d\right) \tan \beta=50, M_{0}=1500 \mathrm{GeV}$. The horizontal lines denote the $2 \sigma$ region of WMAP.

\section{5 $\quad M_{1}^{\prime}>M_{2}^{\prime}$, the case $M_{1}^{\prime}=1.8 M_{2}^{\prime}=1.8 M_{3}^{\prime}$}

We first consider the special case $M_{1}^{\prime}=1.8 M_{2}^{\prime}$. This type of model can arise from string theory. For example in models where gaugino masses are loop suppressed and nonuniversal one loop contribution become dominant, models have been constructed where at the one-loop level the mass $M_{1}^{\prime}$ exceeds $M_{2}^{\prime}$ and $M_{3}^{\prime}$ [94, 95]. We recall that at the electroweak scale this model corresponds to $M_{1} \approx M_{2}$. The lightest neutralino while still dominantly gaugino has a large wino component and is typically heavier than in mSUGRA. Thus the increased importance of the neutralino annnihilation into $W^{+} W^{-}$. Furthermore the LSP can be nearly degenerate with the chargino and $\tilde{\chi}_{2}^{0}$. This favours the gaugino coannihilation channels. The main channels are into quarks and gauge bosons, $\tilde{\chi}_{1}^{0} \tilde{\chi}_{1}^{0}, \tilde{\chi}_{1}^{0} \tilde{\chi}_{2}^{0}, \tilde{\chi}_{1}^{+} \tilde{\chi}_{1}^{-} \rightarrow W^{+} W^{-}$and $\tilde{\chi}_{1}^{0} \tilde{\chi}_{1}^{+} \rightarrow q \bar{q}, Z W$. The allowed region is not anymore restricted to the low $M_{0}$ region as even when squarks and sleptons are very heavy so that the fermion annihilation channels becomes small the annihilation into gauge bosons still occurs and has a large enough rate. In fact this mode is so efficient that this type of model often leads to a value for the relic density that is too low. For example for $\tan \beta=10$, in Fig. 14, the whole region to the left of the $\Omega h^{2}=.094$ contour has a relic density below the WMAP range. Agreement with WMAP then implies a rather heavy LSP, for example $m_{\tilde{\chi}_{1}^{0}} \approx 600 \mathrm{GeV}$ for $\tan \beta=10$.

For neutralinos up to roughly $M_{1 / 2}=700 \mathrm{GeV}$, the coannihilation with the $\tilde{\tau}_{1}$ implies a relic density below the WMAP range. Above this value one does not find an important coannihilation region. The region between the stau LSP border and the $\Omega h^{2}=.129$ 
contour features too high a value for the relic density. There the NLSP is a slepton but slepton coannihilation exchange is not efficient enough for very heavy LSP as we have seen in the mSUGRA case. Since, for a given $M_{1 / 2}$ the LSP is much heavier than in mSUGRA, the scale is sligthly misleading. As one increases $M_{0}$ and the mass of the chargino becomes more degenerate with the mass of the neutralino then the coannihilation with chargino becomes significant and there is a region that satisfies WMAP, in this region one is close to the heavy Higgs pole. The kink at large $M_{1 / 2}$ occurs when the coannihilation process $\tilde{\chi}_{1}^{0} \tilde{\chi}_{1}^{+} \rightarrow t \bar{b}$ lies near the charged Higgs resonance. For large $\tan \beta$, the heavy Higgs pole moves towards lower $M_{1 / 2}$ and merges with the bulk region as well as the focus point region.

The constraints on this type of models from the Higgs mass as well as from precision measurements are not significantly different than in the universal model. For example, the allowed region starts roughly from $M_{1 / 2}>300(450) \mathrm{GeV}$ for $\tan \beta=10(50)$. The first bound is set from the Higgs mass, the second from $b \rightarrow s \gamma$. Note that the Higgs mass and the $b \rightarrow s \gamma$ constrain only models where the relic density is below WMAP.

For collider searches, the spectrum of sparticles as concerns the coloured sector is not dramatically different then in mSUGRA. However since the WMAP allowed regions are completely different and in particular include a Higgs funnel at small tan $\beta$, models with very heavy squarks are perfectly acceptable. For example, for $M_{0}=M_{1 / 2}=2 \mathrm{TeV}$ and $\tan \beta=10$, all squarks are in the $3-4 \mathrm{TeV}$ range. These are beyond the reach of LHC. Furthermore, the LSP tends to be much heavier than in mSUGRA, more importantly, there is a small mass difference between the chargino and the neutralino which can effect searches.

Had we increased further the ratio $r_{12}$ we would have found a LSP with an even larger wino component. This would increase the values for the LSP masses compatible with WMAP. In this way one can easily reconcile the relic density constraint even with a very heavy SUSY spectrum. For example if $r_{12}=2.5$ the LSP as well as the whole supersymmetric spectrum lies above $1.6 \mathrm{TeV}$.

\section{6 $\quad M_{1}^{\prime}<M_{2}^{\prime}=M_{3}^{\prime}$}

In that case, the neutralino can be very light as the constraint on the chargino implies a lighter neutralino than in mSUGRA. A general analysis of MSSM models with light neutralinos and including all constraints has shown that neutralinos as light as $6 \mathrm{GeV}$ could be allowed provided the heavy Higgses were rather light [26, 27]. In SUGRA type models with non-universal gaugino masses however the heavy Higgs mass is not an arbitrary parameter but is deduced from the RGE. Typically one finds a rather heavy scalar, so one cannot appeal to s-channel annihilation through a heavy Higgs resonance to bring the relic density in the desired range, mainly the light Higgs exchange is available. As an example take $M_{1}^{\prime} / M_{2}^{\prime}=1 / 3$, the main annihilation channel is into a pair of fermions. One finds, in addition to the $\tilde{\tau}$ coannihilation region, a region allowed by the relic density constraint, that corresponds to values of $M_{1 / 2}$ where the neutralino can annihilate through a light Higgs pole. This region is compatible with the light Higgs mass limit which is more or less the same as in universal models. For large $\tan \beta$, as in universal models, annihilation into fermions receives a contribution from the heavy Higgs exchange but this is not sufficient to allow very light neutralinos. The relic density constrains neutralinos to be heavier than 

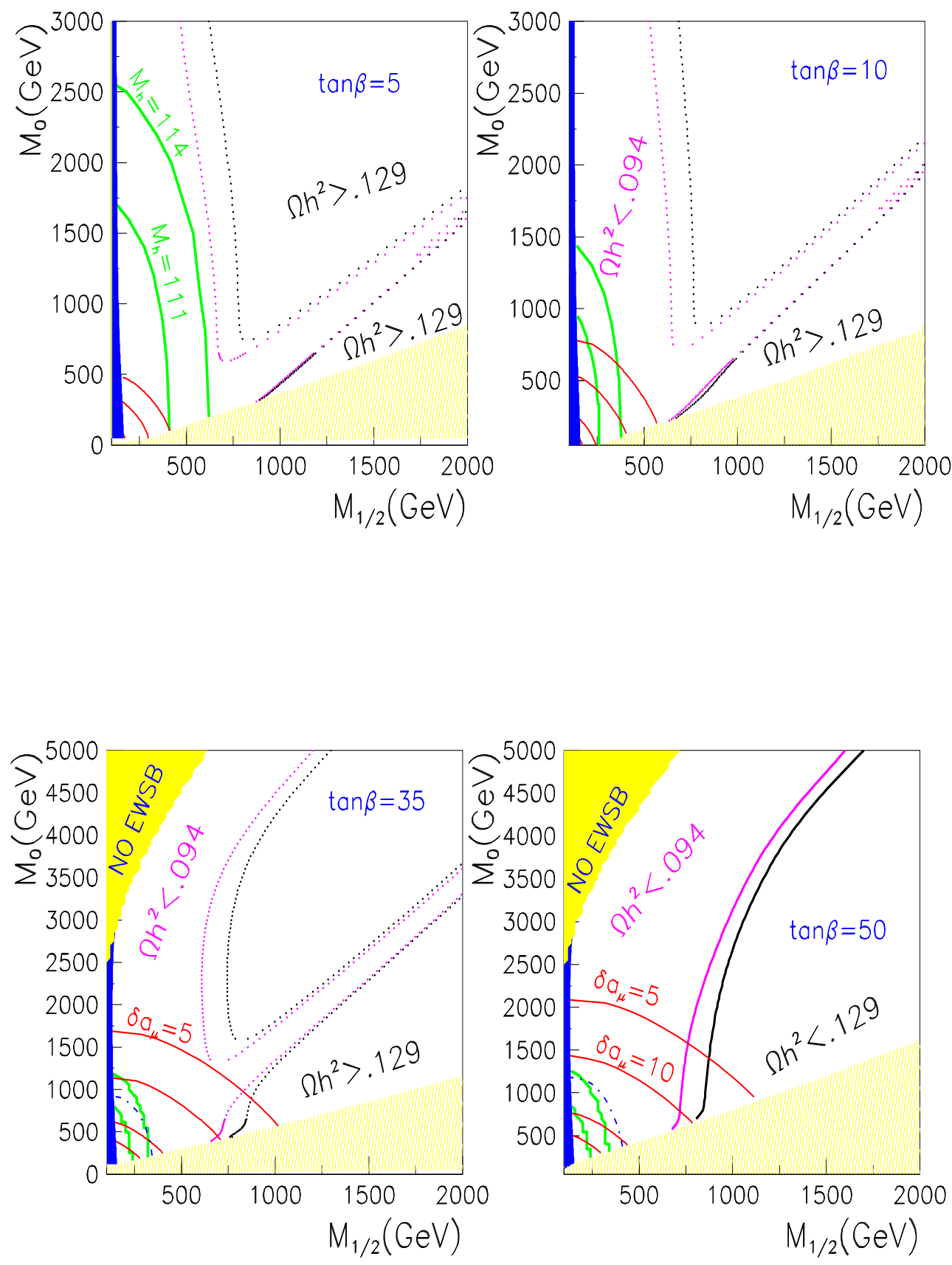

Figure 14: Same as Figure 10 for $\mu>0, A_{0}=0, m t=175 \mathrm{GeV}, M_{1}^{\prime}=1.8 M_{2}^{\prime}=1.8 M_{3}^{\prime}$. 
$m_{\tilde{\chi}_{1}^{0}} \approx 30 \mathrm{GeV}$. This type of model offers good prospect for direct detection of the LSP as well as for supersymmetry discovery both at the LHC and at the linear collider as was discussed in [26, 27].

\subsection{Other non-universal cases}

Finally there is another class of models that feature non-universal gaugino masses, models with $M_{1}^{\prime}=M_{3}^{\prime} \neq M_{2}^{\prime}$ 93, 91, 96. We do not discuss these models here as the main impact of relaxing universality has already been illustrated in the cases studied above in details. First we expect that when $M_{1}^{\prime}=M_{3}^{\prime}>M_{2}^{\prime}$ the relic density would be easily satisfied because of the large wino component. Of course, as compared to the case where $M_{3}^{\prime}=M_{2}^{\prime}$ discussed above, shifts in the value of $m_{A}$ and $\mu$ will result from an increase in $M_{3}^{\prime}$. Second we expect models with $M_{1}^{\prime}=M_{3}^{\prime}<M_{2}^{\prime}$, to be rather similar to the $M_{3}^{\prime}<M_{2}^{\prime}$ models since the crucial parameters for the evaluation of the relic density, such as $\mu$ and $M_{A}$ are determined by $M_{3}^{\prime}$ via the renormalization group equations.

\section{Prospects for direct detection}

We have just shown that cosmological measurements provide strong constraints on many supersymmetric models. Here we discuss the potential of direct detection searches for the LSP both in the mSUGRA model and non-universal models.

Several experiments have placed limits on the cross section for the spin independent neutralino proton scattering, typically at the level of $10^{-6} \mathrm{pb} 34$. There is also one report of a signal by DAMA 35. In the near future results form several upgraded detectors are expected. In fact, the first results from CDMS2 have been published recently 36] showing already significant improvement on the upper limit. Finally ton-scale detectors are also planned, such as ZeplinIV[37, Genius 38 and Xenon[39]. These large scale detector will reach a sensitivity between $10^{-9}-10^{-10} \mathrm{pb}$ for neutralinos around $100 \mathrm{GeV}$. Typically detectors have a reduced sensitivity as one increases the neutralino mass. In parallel, detectors that are also sensitive to the spin dependent part of the neutralino proton cross section have obtained their first results, Naiad 40, Simple 41] and PICASSO 42. At the moment these detectors are much less precise and typically limits at the level of a few pb's are given. Upgrades of these detectors are planned and eventually a ton-size detector such as PICASSO could reach a sensitivity between $10^{-5}-10^{-4} \mathrm{pb}$.

We follow the usual practice and use the cross section for annihilation of neutralino on proton, $\sigma_{\tilde{\chi}_{1}^{0} p}$, to compare the direct detection potential in a given model. We do not rescale the cross section to take into account the possibility that the local density of neutralino could be lower than expected. However one has to keep this point in mind especially for the models where the relic density is very low. The spin-independent cross section is dominated by the Higgs exchange and squark exchange diagrams whereas the spin dependent one receives contribution from the $\mathrm{Z}$ exchange and squark exchange diagrams. One therefore expects to find the largest cross sections, for both spin-dependent and spinindependent processes, in the low $M_{0}$ region where light squarks are found as well as in the focus point region of mSUGRA models [97] where the coupling of neutralinos to both the light Higgs and the $\mathrm{Z}$ are enhanced because of the mixed bino/Higgsino nature of the 
neutralino. Note that in the focus point region, the large coupling to the Z/Higgs implies a large annihilation cross sections of neutralinos as well as large neutralino proton cross sections.

\section{1 mSUGRA}

We have performed scans over the parameter space of the mSUGRA model with $\mu>0$, $A_{0}=0, M_{0}<5 \mathrm{TeV}$ and $M_{1 / 2}<2 \mathrm{TeV}$ for $\tan \beta=10$ and $\tan \beta=50$ as in previous sections. We first consider a top quark mass $m_{t}=175 \mathrm{GeV}$. While the prediction for the neutralino proton cross sections vary over several orders of magnitude over the full parameter space, after imposing the WMAP upper bound one distinguishes easily the stau coannihilation region, the light Higgs annihilation region and the focus point region. In the latter case the cross sections are typically larger despite the suppressed contribution of the squark exchange. The reason is the enhanced coupling of the neutralino to the Higgs due to the mixed Higgsino/gaugino nature of the neutralino. Note that the same mechanism that made for efficient neutralino annihilation also induces large values for the spin independent cross section. As discussed previously, the focus point region is found only at large $\tan \beta$ in mSUGRA models for $m_{t}=175 \mathrm{GeV}$. In Fig. 15 the focus point region for $\tan \beta=50$ corresponds to the set of points clustered around $m_{\tilde{\chi}_{1}^{0}}=300 \mathrm{GeV}$ for which $\sigma_{S I} \approx 1-3 \times 10^{-8} \mathrm{pb}$. This region can then be partly probed by EdelweissII and will be completely covered by future detectors such as ZeplinIV. In general, one also gets the contribution of the squark exchange diagram. Thus large cross sections are also expected in the low $M_{0}$ region of parameter space where one finds the lighter squarks, this basically means the stau coannihilation region. Furthermore, the spin-independent cross section should increase for lighter neutralinos as they are found in the low $M_{0}-M_{1 / 2}$ region where in addition to having light squarks the coupling of the neutralino to the Higgs is large as well. Note however that this is precisely where the collider constraints come into play. After imposing all constraints we find the maximum value $\sigma_{S I}<3 \times 10^{-9}\left(10^{-8}\right) \mathrm{pb}$ for $\tan \beta=10(50)$. These can only be partially probed by future large scale detectors. For example, Xenon will probe models for which $m_{\tilde{\chi}_{1}^{0}}<250(360) \mathrm{GeV}$.

At large $\tan \beta$ there is also a region where neutralinos annihilate via a heavy Higgs exchange, in this region, neutralinos are heavy as well as the squarks and the heavy Higgs which lie in the $\mathrm{TeV}$ range. One therefore does not expect a large cross section despite the enhanced coupling of the Higgs to bottom quarks. Indeed this region features cross sections $\mathcal{O}\left(10^{-10}\right) \mathrm{pb}$ and shows up as the continuation of the coannihilation region for $m_{\tilde{\chi}_{1}^{0}} \approx 500 \mathrm{GeV}$ in Fig. 15. Finally the region at intermediate $M_{0}$ where it is still possible to have annihilation of neutralinos via the exchange of a light Higgs scalar predict values within the range $10^{-10}\left(10^{-9}\right)<\sigma_{S I}<10^{-9}\left(10^{-8}\right)$ pb for $\tan \beta=10(50)$. The enhancement at large $\tan \beta$ is due to the heavy Higgs contribution. While the heavy Higgs exchange is completely negligible at intermediate values of $\tan \beta$ it eventually dominates the light Higgs despite the mass suppression factor. This is due partly to a decrease of the mass of the Heavy Higgs in models with large $\tan \beta$ but mainly one benefits from the enhanced couplings of the heavy Higgs to bottom quarks. This region is accessible to future large scale detectors such as Xenon 39 .

The spin dependent cross section has roughly the same behaviour as the spin independent one. Indeed the neutralino LSP couplings to the Z is also enhanced in the focus point region because of the larger Higgsino component. This region corresponds to the 
sest of points clustered around $\sigma_{S D} \approx 10^{-4} \mathrm{pb}$ in Fig [15] and can be probed entirely by future large scale detectors such as PICASSO 42. In the coannihilation region and the heavy Higgs annihilation region, the cross section decreases with an increase of the neutralino mass. As above this is related to the additional suppression of the heavy squark exchange. The predictions for the spin dependent cross sections still lie orders of magnitude below present and future limits especially in the heavy Higgs annihilation region at $\tan \beta=50$ where $\sigma_{S D} \approx 10^{-8} \mathrm{pb}$. Finally the allowed models with light neutralinos for which a good relic density is found since neutralino annihilates via the light Higgs have also an enhanced coupling to the $\mathrm{Z}$. Here one finds rather favourable cross sections, $\sigma_{S I} \approx 10^{-4}-10^{-5}$. These models can be probed by a ton-scale detector.

The neutralino proton cross sections should not depend very much on the value of the top quark mass for a given set of MSSM parameters. However the allowed parameter space and the predictions for the MSSM parameters within the mSUGRA model differs significantly as discussed in section 3. If we increase the top quark mass, $m_{t}=179 \mathrm{GeV}$, the focus point region will be shifted to higher values of $M_{0}$, out of the range scanned here. Furthermore the heavy Higgs annihilation region almost disappeared. We then recover the results for the spin independent cross section for $m_{t}=175 \mathrm{GeV}$ in the coannihilation and Higgs annihilation regions. However since the Higgs mass constraint is relaxed one can reach higher cross sections for light neutralinos, for example $\sigma_{S I} \approx 5 \times 10^{-8} \mathrm{pb}$ for $\tan \beta=10$. For $\tan \beta=50$ the maximum cross section in the coannihilation region is similar to the case of the lighter top quark since the constraints from colliders are much less dependent on the top quark mass.

We also discuss the case of a rather light top quark since WMAP allows a more important region of parameter space and we can better illustrate the generic behaviour of the neutralino proton cross section in the different regions of mSUGRA models. The results of a scan over models with $m_{t}=170 \mathrm{GeV}$ are displayed in Fig. 15. The focus point region with a Higgsino LSP is allowed even for $\tan \beta=10$, and as above that is where one finds the largest cross sections, $\sigma_{S I} \approx 10^{-8}-10^{-7} \mathrm{pb}$ over the allowed range of neutralino masses. Much lower cross sections are found in the coannihilation region. For $\tan \beta=50$, the allowed region covers most of the parameter space (see Fig 5 b). Nevertheless one can roughly distinguish the focus point, coannihilation and Higgs annihilation regions in Fig 16b. The largest cross sections are again found in the focus point region where for light neutralinos $\sigma_{S I}$ can reach $10^{-7} \mathrm{pb}$. The maximum value for the spin-independent cross section increases with $\tan \beta$ since one finds a neutralino LSP with a higher Higgsino content. In the coannihilation region which shows up as the lower branch in Fig. 16] , the upper limit drops to $\sigma_{S I}<10^{-8} \mathrm{pb}$ after imposing the constraints from colliders. In the region of the Higgs funnel where neutralinos are above $500 \mathrm{GeV}$, the spin-independent cross section drops to rather low values, $\sigma_{S I}>7 \times 10^{-11} \mathrm{pb}$, beyond the reach of future detectors. Basically the conclusions for the direct detection potential are similar to the ones reached for $m_{t}=175 \mathrm{GeV}$, the best prospects are in the focus point region and for light neutralinos in the coannihilation region while the heavy neutralinos found in either the Higgs funnel or the coannihilation region will remain out of reach.

To summarize the results in the mSUGRA model, the most promising models for both spin independent and spin dependent direct detection are models with a mixed bino/Higgsino LSP. For a top quark mass above $175 \mathrm{GeV}$, the former is expected only at large $\tan \beta$. Models with almost a pure bino LSP predict lower cross sections but should still be within reach of the large scale detectors provided neutralinos do not exceed roughly 

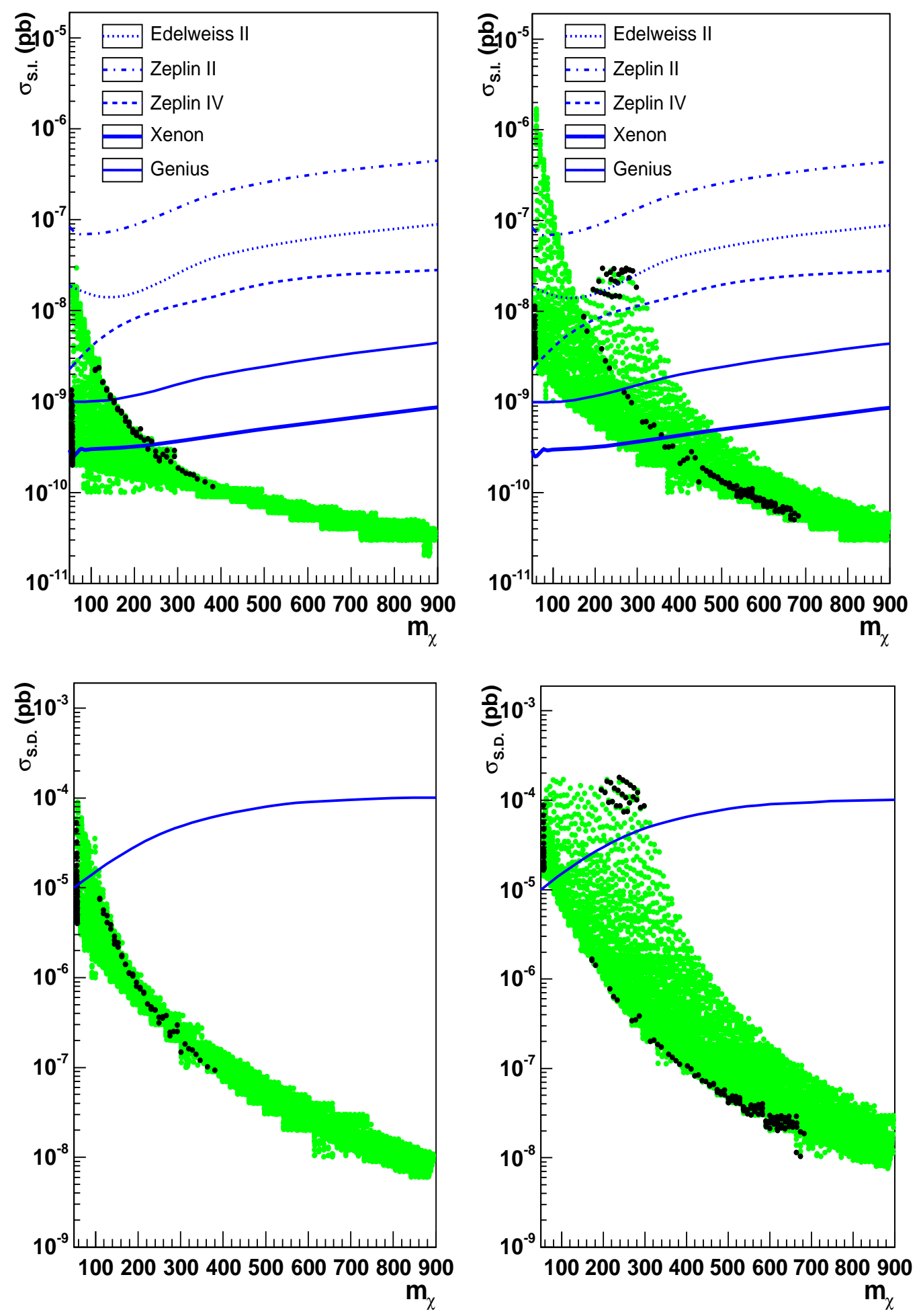

Figure 15: Spin independent (top) and spin dependent (bottom) cross sections for scattering of neutralinos on proton as fonction of $m_{\tilde{\chi}_{1}^{0}}$ for $m_{t}=175 \mathrm{GeV}, \tan \beta=10$ (left) and $\tan \beta=50$ (right). Black dots denoted models for which $\Omega h^{2}<.129$. The line in the bottom figures is the expected limit from PICASSO. 
$400 \mathrm{GeV}$. Models that belongs to the Higgs funnel region cannot be probed even at the large scale detectors.
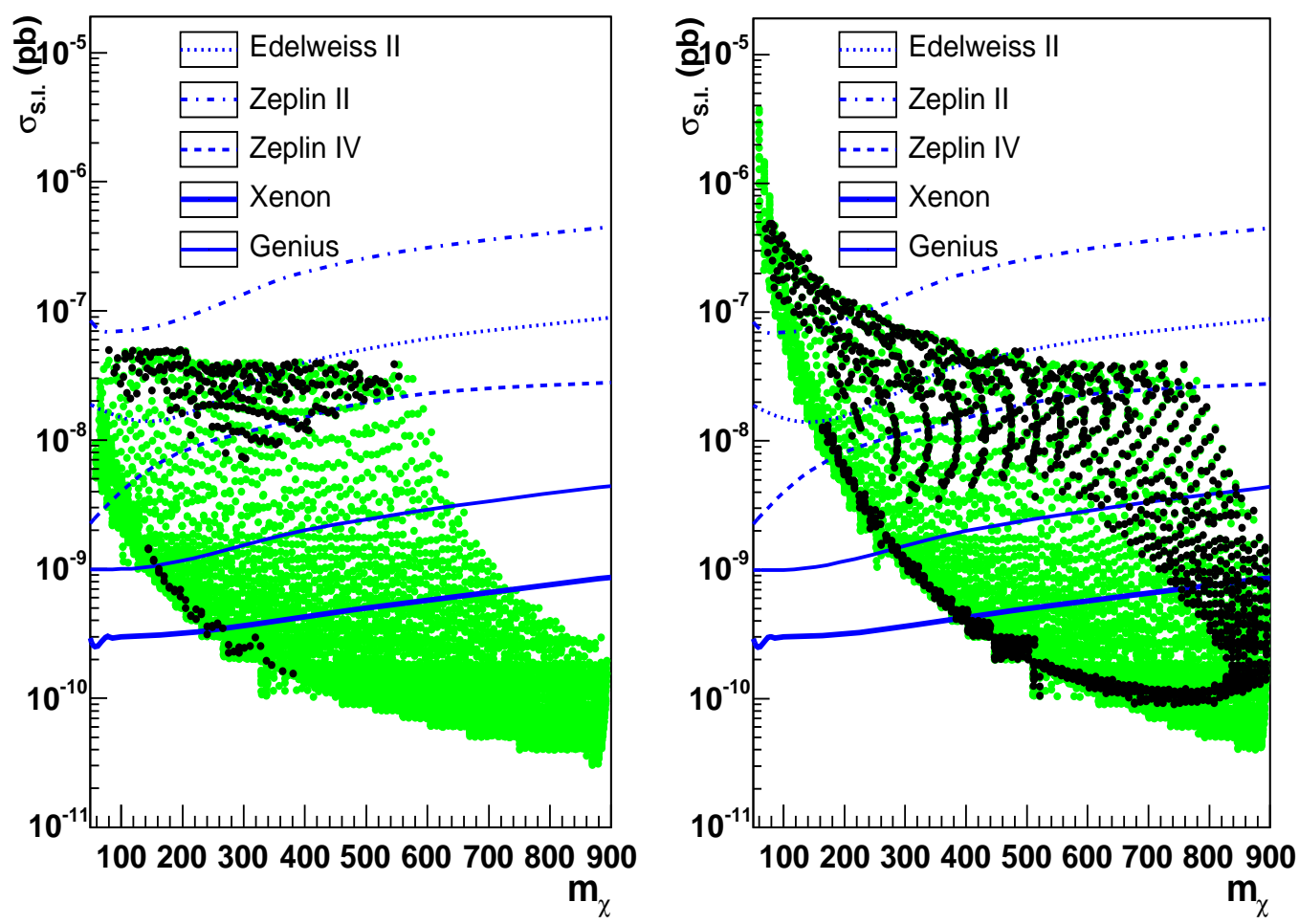

Figure 16: Spin independent cross sections for scattering of neutralinos on proton as fonction of $m_{\tilde{\chi}_{1}^{0}}$ for $m_{t}=170 \mathrm{GeV}, \tan \beta=10$ (left) and $\tan \beta=50$ (right). Black dots denoted models for which $\Omega h^{2}<.129$

\subsection{Non-universal models}

We next discuss non-universal models, considering the two cases that differ significantly from mSUGRA as far as the relic density is concerned: the case with a LSP with a higher Higgsino content, $M_{3}<M_{2}$ as well as the case with a wino LSP, $M_{1}>M_{2}$. Since the LSP coupling to the $\mathrm{Z}$ and/or Higgs is a crucial parameter in evaluating the neutralino proton cross section one expects significant differences with the mSUGRA model.

\subsection{1 $\quad M_{3}^{\prime}=M_{2}^{\prime} / 2$}

First consider the model $r_{32}=2$ and $m_{t}=175 \mathrm{GeV}$. We have performed a scan over the parameters $M_{2}^{\prime}<4 \mathrm{TeV}$ and $M_{0}<5 \mathrm{TeV}$. As we have discussed before, both the squarks and the heavy Higgs tend to be lighter than in universal models. Furthermore light neutralinos have a higher Higgsino component since $\mu$ is typically smaller. Therefore one expects an enhanced scalar cross section. In Fig 17 we indeed see that in the coannihilation region the lower bound on the scalar cross section is around $5 \times 10^{-9} \mathrm{pb}$ and that it can exceed $\sigma_{S I}=10^{-8} \mathrm{pb}$ for $\tan \beta=10$ for light squarks and neutralinos. The coannihilation/heavy Higgs region correspond to the inverted S-shape region in Fig. 17a. The 
enhancement of the cross section relative to the universal model is especially noticeable for heavy neutralinos. This is solely due to the enhanced coupling of the neutralino to the light Higgs. We had found two focus point regions in Fig. 11, In Fig. 17, the first region corresponds to the sets of points around $m_{\tilde{\chi}_{1}^{0}} \approx 200 \mathrm{GeV}$ for which $\sigma_{S I} \approx 10^{-8}$, the second region to $m_{\tilde{\chi}_{1}^{0}} \approx 800-1200 \mathrm{GeV}$. Towards the upper range of this region the cross section drops to $\sigma_{S I} \approx 4 \times 10^{-9}$. As in the focus point region of the universal model, reasonable cross sections are found despite the very heavy squarks because of the mixed bino/Higgsino nature of the LSP. There is finally one region where one finds $\sigma_{S I} \approx 10^{-9} \mathrm{pb}$, there neutralinos are light and annihilate through the light Higgs. We had already found a similar region in mSUGRA with roughly the same cross section. In this model, for $\tan \beta=10$, detectors such as Edelweiss probe part of the focus point region and almost all regions are within reach of future detectors like Genius.

For $\tan \beta=50$, most of the parameter space is allowed by the relic density constraint. The predictions for $\sigma_{S I}$ spans a wide range of values. The largest cross sections are found in the low $M_{0}-M_{1 / 2}$ plane, however once the limit on the Higgs mass and on $b \rightarrow s \gamma$ are taken into account one finds $\sigma_{S I}<8 \times 10^{-8} \mathrm{pb}$. Many models have $\sigma_{S I}<10^{-9} \mathrm{pb}$, the absolute lower bound is $3 \times 10^{-10} \mathrm{pb}$, an enhancement as compared to universal models. This is again directly related to the value of $\mu$ which entails a LSP with a higher Higgsino content than in mSUGRA. Some scenarios can be probed by EdelweissII but only if $m_{\tilde{\chi}_{1}^{0}}<400 \mathrm{GeV}$. To probe a large fraction of the parameter space one has to wait for large detectors like Xenon, even then it will not be possible to cover the full parameter space.
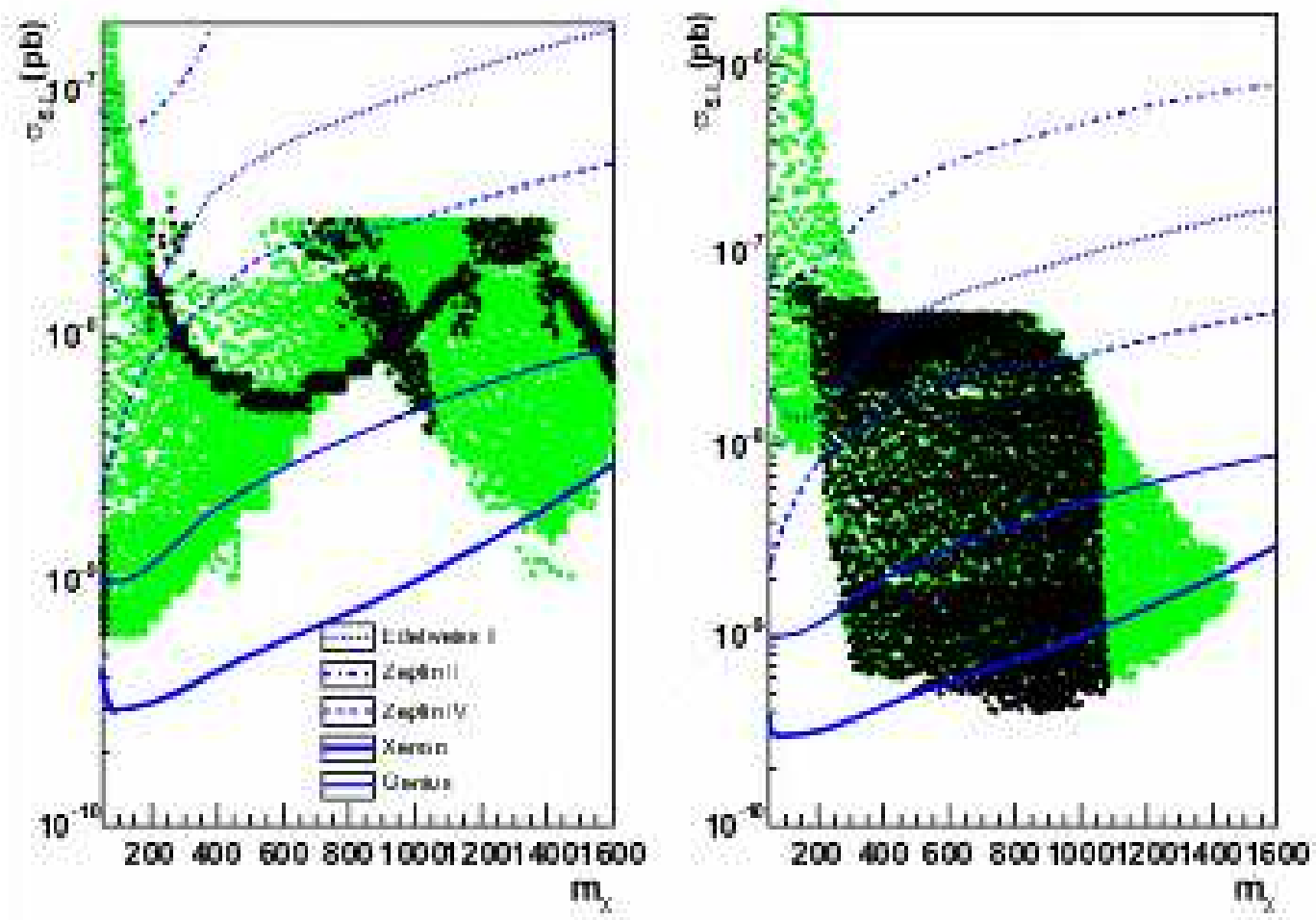

Figure 17: Spin independent (top) cross sections for scattering of neutralinos on proton as fonction of $m_{\tilde{\chi}_{1}^{0}}$ for $\tan \beta=10$ and $\tan \beta=50$ in models where $M_{3}^{\prime}=M_{2}^{\prime} / 2$. 


\section{$\mathbf{5 . 2 . 2} \quad M_{1}^{\prime}=1.8 M_{2}^{\prime}$}

When $M_{1}^{\prime}=1.8 M_{2}^{\prime}$ and the LSP is wino-like, the region where the WMAP upper bound is satisfied includes all models with $m_{\tilde{\chi}_{1}^{0}}<600 \mathrm{GeV}$. There the spin independent cross section ranges from $\sigma_{S I} \approx 10^{-9}-5 \times 10^{-8} \mathrm{pb}$ for $\tan \beta=10$. For a given neutralino mass, the larger values for the cross sections, as usual, correspond to the lighter squarks. For heavier neutralinos, the WMAP allowed region is one of gaugino coannnihilation and heavy Higgs annihilation. Although coannihilation helps reduce the relic density it does not impact directly on the neutralino proton cross section, one finds small cross sections which further decrease as one increases $m_{\tilde{\chi}_{1}^{0}}$. Note that for a fixed value of the neutralino mass in the coannihilation/Higgs funnel region, the points that are allowed by WMAP have the lowest direct detection cross section since they correspond to larger values of $M_{0}$. For $\tan \beta=10$, detectors like EdelweissII can probe some of the models but even with Xenon, models with heavy neutralinos cannot be probed. For $\tan \beta=50$, the prospects for detection by large scale detectors are better, covering all the allowed parameter space. The main reason is that models with heavy neutralinos can only be found in the focus point region where the Higgs and $\mathrm{Z}$ exchange contribution is important.
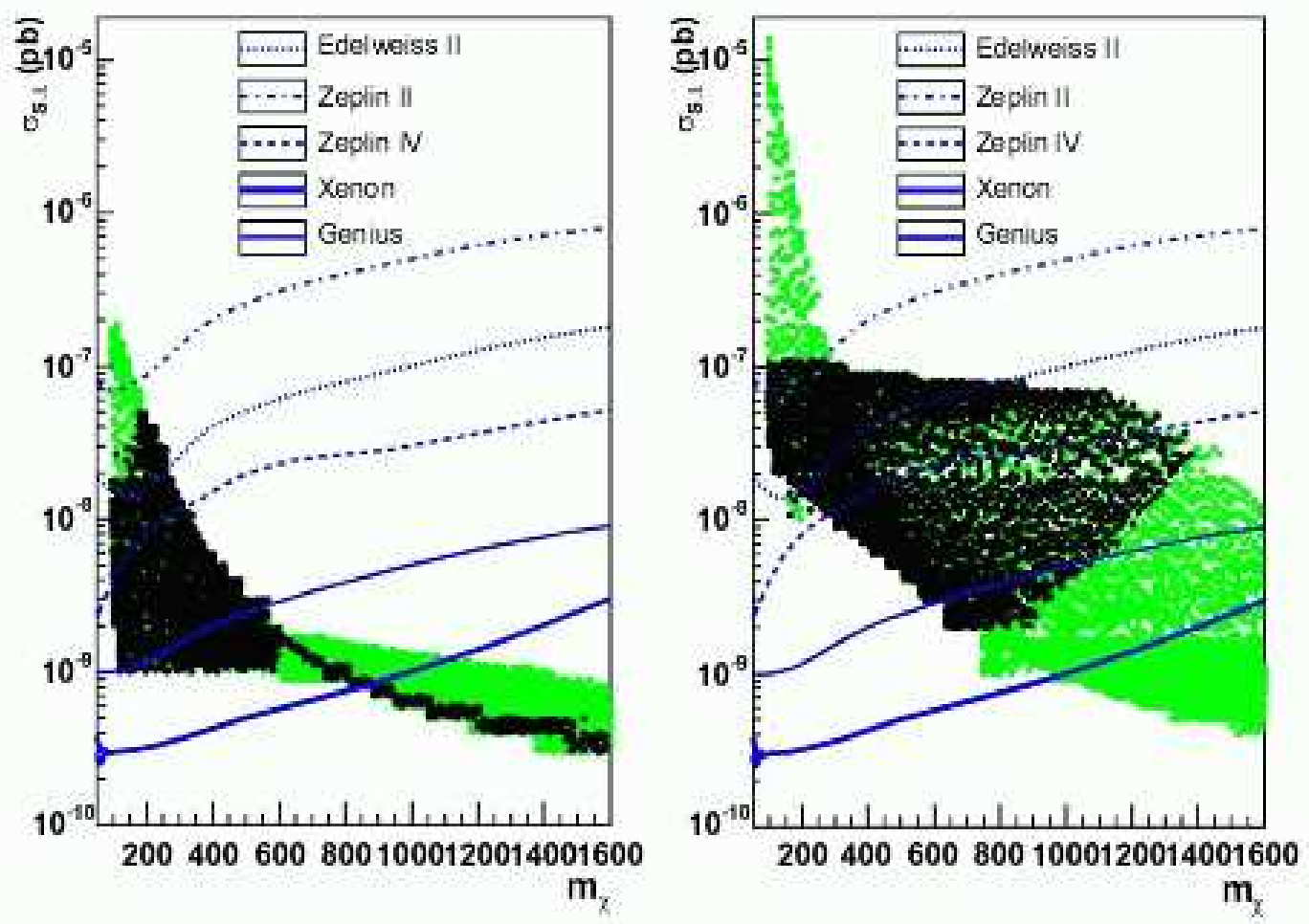

Figure 18: Spin independent cross sections for scattering of neutralinos on proton as fonction of $m_{\tilde{\chi}_{1}^{0}}$ for $\tan \beta=10$ and $\tan \beta=50$ in models where $M_{1}^{\prime}=1.8 M_{2}^{\prime}$.

\subsection{Summary}

In summary in the focus point region of mSUGRA the prospects are good for direct detection of dark matter by the next generation of detectors such as EdelweissII. In the stau coannihilation region the situation is more difficult and one will have to wait for large 
scale detectors such as Xenon. Even then only a fraction of the parameter space can be probed. The Higgs funnel region is extremely dificult even for the most ambitious project as this region is also characterized by heavy neutralinos and rather heavy squarks, one has to face the problem of reduced sensitivity of detectors even when the cross section is not suppressed significantly. In models with non-universal gaugino masses $M_{3}<M_{2}$, prospects are better since one finds larger values for the neutralino couplings to the $\mathrm{Z}$ and the light Higgs. The lighter squarks also tend to increase the cross sections for a given neutralino mass. In models where $M_{1}>M_{2}$ also the prospects for direct detection are more promising except in the coannihilation/Higgs funnel region at low $\tan \beta$. At large $\tan \beta$ the full parameter space can be basically covered since the models with heavy neutralinos and squarks that are hard to probe are excluded.

\section{Conclusion}

We have presented constraints on both mSUGRA and non-universal gaugino masses models emphasizing the role of the latest measurements of WMAP on the relic density of dark matter. Our calculations are based on micrOMEGAs coupled to SOFTSUSY for the evaluation of the spectrum.

We found, in agreement with previous analyses, that the new results of WMAP have almost excluded the bulk region at low $M_{0}-M_{1 / 2}$ in mSUGRA and that it imposes very specific relation among sparticle masses and parameters:

- Neutralino nearly degenerate with a slepton,

- Neutralino mass near half the mass of a Higgs,

- Neutralino with a significant Higgsino component.

The latter is found in the focus point region although the location of the focus point region depends strongly on the value of the top quark mass and to a lesser extent on the choice of $A_{0}$. This comes as no surprise since the solution of the RG equation for $\mu$ are very sensitive to the top Yukawa coupling. Furthermore the different implementations of the higher loop corrections in the RGE means that there are still differences between the predictions of different codes. In our case we found that a top quark mass above $179 \mathrm{GeV}$ pushes the focus point above $M_{0}=5 \mathrm{TeV}$. One is then basically left with the coannihilation region as well as a Higgs annihilation region. The latter includes a possibility for annihilation near a light Higgs resonance but annihilation through a heavy Higgs resonance is more likely to occur at large $\tan \beta$. Conversely if the top quark mass lies towards the lower end of the allowed range, a large focus point region is cosmologically allowed even at low $\tan \beta$. On the other hand the constraint from the light Higgs mass is more severe especially at low values of $\tan \beta$. Note that regions where the stop is the NLSP are usually ruled out by direct constraints, still the possibility exists in models with a large mixing in the stop sector.

These conclusions result, in some sense, from the relation between the parameters of the MSSM obtained in the mSUGRA model. In order to assess how general the statements about the importance of the relic density constraints are, we have moved 
away from mSUGRA by introducing some non-universality in the gaugino sector. By doing so one can deviate from mSUGRA predictions for the parameters that are critical in the evaluation of the relic density in particular the value of $\mu$. This parameter in relation to $M_{1}, M_{2}$ determines the gaugino/Higgsino content of the LSP and as a result directly the annihilation cross sections into fermions and gauge bosons. The impact of this parameter is already visible in mSUGRA where as soon as one could get away from the pure bino case, one finds (in the focus point region) much more efficient annihilation of neutralinos. In non-universal MSSM we have, in addition to a Higgsino LSP, models with a wino LSP. In these models the upper bound on the relic density can easily be satisfied, because of the large cross section for the annihilation of neutralinos into $\mathrm{W}$ pairs. These models are the ones where $M_{1}^{\prime}>M_{2}^{\prime}$ at the GUT scale. Second, by changing the relation between the heavy Higgs mass and the neutralino mass we have found that annihilation through a heavy Higgs exchange can take place even at low $\tan \beta$. These conditions can be realized in non-universal gaugino mass models, by changing the mass relation between $M_{3}^{\prime}$ and $M_{2}^{\prime} / M_{1}^{\prime}$. Indeed $M_{A}$ is sensitive to the value of $M_{3}^{\prime}$ at the high scale. Finally in all models we found a region where the WMAP upper limit could be satisfied because of the contribution of slepton coannihilation channels as long as the neutralino and stau were not too heavy. In summary in non-universal gaugino mass models, compatibility with the WMAP measurement imposes similar conditions on the supersymmetric masses and parameters than in mSUGRA. The only new possibility is for a wino LSP in addition to the Higgsino LSP. We stress that SUGRA models with non-universal gaugino masses give a good picture of the dark matter constraints in the general MSSM while keeping with a reasonable number of free parameters. Although we have mainly studied only two non-universal gaugino mass models, it was sufficient to illustrate the main mechanism at work in the relic density.

We have also discussed the potential for probing SUGRA models with the different experiments for direct detection of the LSP. While few models lead to prediction for the direct annihilation cross section within reach of the current experiments, a large fraction of the parameter space will be probed by ton-scale future detectors. Typically models with a Higgsino LSP predict the largest cross sections while coannihilation models feature much lower cross sections, especially when the sfermion masses increase. In fact the Higgsino component of the LSP is also what makes efficient annihilation of neutralino pairs and leads to acceptable values for the relic density. Finally when the neutralino annihilates via a heavy Higgs, few models can lead to a signal for direct detection especially when they feature a heavy LSP. We keep a more detailed comparative study of the direct detection and collider searches for a future publication.

\section{Acknowledgements}

We thank B. Allanach for discussions and help with the SOFTSUSY code. This work was supported in part by the PICS-397 of CNRS, Calcul en physique des particules, by GDRI-ACPP of CNRS and by grants from the Russian Federal Agency for Science, NS1685.2003.2 and RFBR 04-02-17448. 


\section{References}

[1] D. N. Spergel et. al., Astrophys. J. Suppl. 148 (2003) 175, astro-ph/0302209.

[2] C. L. Bennett et. al., Astrophys. J. Suppl. 148 (2003) 1, astro-ph/0302207.

[3] H. Baer et. al., JHEP 07 (2002) 050, hep-ph/0205325.

[4] J. R. Ellis, K. A. Olive, Y. Santoso, and V. C. Spanos, Phys. Lett. B565 (2003) 176-182, hep-ph/0303043.

[5] U. Chattopadhyay, A. Corsetti, and P. Nath, Phys. Rev. D68 (2003) 035005, hep-ph/0303201.

[6] A. B. Lahanas and D. V. Nanopoulos, Phys. Lett. B568 (2003) 55-62, hep-ph/0303130.

[7] H. Baer, C. Balazs, and A. Belyaev, JHEP 03 (2002) 042, hep-ph/0202076.

[8] A. Djouadi, M. Drees and J. L. Kneur, JHEP 0108 (2001) 055, hep-ph/0107316; L. Roszkowski, R. Ruiz de Austri and T. Nihei, JHEP 0108 (2001) 024, hep-ph/0106334;

J. R. Ellis, T. Falk, G. Ganis, K. A. Olive and M. Srednicki, Phys. Lett. B510(2001) 236.

[9] J. Ellis, T. Falk, G. Ganis and K. A. Olive, Phys. Rev. D62:075010 (2000); hep-ph/0004169 J. Ellis, G. Ganis, D. V. Nanopoulos, K. A. Olive and M. Srednicki, Phys. Lett. B510(2001) 236; hep-ph/0102098

[10] H. Baer and C. Balazs, JCAP 0305 (2003) 006, hep-ph/0303114.

[11] M. Drees and M. Nojiri, Phys. Rev. D47 (1993) 376

R. Arnowitt and P. Nath, Phys. Lett. B299(1993) 58.

[12] K. Griest and D. Seckel, Phys. Rev. D43 (1991) 3191-3203.

[13] A. Birkedal-Hansen and E.-h. Jeong, JHEP 02 (2003) 047, hep-ph/0210041.

[14] J. R. Ellis, K. A. Olive, Y. Santoso, and V. C. Spanos, Phys. Rev. D69 (2004) 095004, hep-ph/0310356.

[15] A. Romanino and A. Strumia, Phys. Lett. B487 (2000) 165-170, hep-ph/9912301.

[16] B. C. Allanach, S. Kraml, and W. Porod, JHEP 03 (2003) 016, hep-ph/0302102.

[17] J. Ellis, K. Enqvist, D.V. Nanopoulos and K. Tamvakis, Phys. Lett. B155 (1985) 381.

M. Drees, Phys. Lett. B158 (1985) 409; Phys. Rev. D33 (1986) 1486.

[18] A. Brignole, L.E. Ibáñez, C. Muñoz, Nucl. Phys. B422 (1994) 125, Erratum B436 (1995) 747, hep-ph/9308271. For a review see A. Brignole, L.E. Ibáñez, C. Muñoz, hep-ph/9707209. 
[19] T. Kobayashi, D. Suematsu, K. Yamada and Y. Yamagishi, Phys. Lett. B348 (1995) 402, hep-ph/9408322.

A. Brignole, L.E. Ibáñez, C. Muñoz and C. Scheich, Zeit. Phys. C74 (1997) 157.

hep-ph/9508258.

Y. Kawamura, S. Khalil and T. Kobayashi, Nucl. Phys. B502 (1997) 37,

hep-ph/9703239 and references therein.

[20] T. Gherghetta, G.F. Giudice and J.D. Wells, Nucl. Phys. B559 (1999) 27,

hep-ph/9904378.

Z. Chacko, M.A. Luty, I. Maksymyk and E. Ponton, JHEP 0004 (2000) 001, hep-ph/9905390.

M.A. Luty and R. Sundrum, Phys. Rev. D62 (2000) 035008, hep-th/9910202.

J.A. Bagger, T. Moroi and E. Poppitz, JHEP 0004 (2000) 009, hep-th/9911029.

R. Rattazzi, A. Strumia and J.D. Wells, Nucl. Phys. B576 (2000) 3, hep-ph/9912390.

[21] G. F. Giudice, M. Luty, H. Murayama and R. Rattazzi, JHEP 9812 (1998) 027.

L. Randall and R. Sundrum, Nucl. Phys. B557 (1999) 79.

A. Pomarol and R. Rattazzi, JHEP 9905(1999) 013.

K. Huitu, J. Laamanen and P. N. Pandita, Phys.Rev. D65 (2002) 115003, hep-ph/0203186.

[22] C. H. Chen, M. Drees, and J. F. Gunion, Phys. Rev. D55 (1997) 330-347, hep-ph/9607421.

[23] P. Binétruy, A. Birkedal-Hansen, Y. Mambrini, and B. D. Nelson, hep-ph/0308047.

[24] A. Birkedal-Hansen and B. D. Nelson, Phys. Rev. D64 (2001) 015008, hep-ph/0102075.

[25] G. Bélanger, F. Boudjema, A. Pukhov, and S. Rosier-Lees, hep-ph/0212227.

[26] G. Bélanger, F. Boudjema, A. Cottrant, A. Pukhov, and S. Rosier-Lees, JHEP 03 (2004) 012, hep-ph/0310037.

[27] A. Bottino, F. Donato, N. Fornengo, and S. Scopel, Phys. Rev. D68 (2003) 043506, hep-ph/0304080.

[28] H.N. Brown et al., Muon g-2 Collaboration, Phys. Rev. Lett. 86 (2001) 2227;

hep-ex/0102017.

G.W. Bennett et al., Muon g-2 Collaboration, Phys. Rev. Lett. 89 (2002) 101804,

Erratum-ibid 89 (2002) 1219903, hep-ex/0208001.

[29] M. Davier, S. Eidelman, A. Hocker, and Z. Zhang, Eur. Phys. J. C31 (2003)

503-510, hep-ph/0308213.

[30] B. C. Allanach, Comput. Phys. Commun. 143 (2002) 305-331, hep-ph/0104145.

[31] B. C. Allanach, G. Bélanger, F. Boudjema, A. Pukhov, and W. Porod, hep-ph/0402161.

[32] B. Allanach, G. Bélanger, F. Boudjema, A. Pukhov, in preparation; G. Bélanger, talk given at LCWS, Paris, april 2004. 
[33] G. Gelmini, P. Gondolo, hep-ph/0405278

[34] V. Sanglard, the EDELWEISS Collaboration, astro-ph/0306233.

[35] The DAMA Collaboration, R. Bernabei et al., Riv. N. Cim. 26 n.1 (2003) 1; astro-ph/0307403.

[36] D. S. Akerib et. al.,, CDMS Collaboration astro-ph/0405033.

[37] R. Luscher, astro-ph/0305310.

[38] H. V. Klapdor-Kleingrothaus and I. V. Krivosheina, prepared for DARK 2002: 4th International Heidelberg Conference on Dark Matter in Astro and Particle Physics, Cape Town, South Africa, 4-9 Feb 2002.

[39] E. Aprile et. al., astro-ph/0207670.

[40] B. Ahmed et. al., Astropart. Phys. 19 (2003) 691-702, hep-ex/0301039.

[41] F. Giuliani and T. A. Girard, Phys. Lett. B588 (2004) 151-154, astro-ph/0311589.

[42] N. Boukhira et. al., Nucl. Phys. Proc. Suppl. 110 (2002) 103-105.

[43] G. Bélanger, F. Boudjema, A. Pukhov, and A. Semenov, hep-ph/0405253.

[44] G. Bélanger, F. Boudjema, A. Pukhov, and A. Semenov, Comput. Phys. Commun. 149 (2002) 103-120, hep-ph/0112278.

[45] P. Skands et. al., hep-ph/0311123.

[46] J. R. Ellis, T. Falk, K. A. Olive, and Y. Santoso, Nucl. Phys. B652 (2003) 259-347, hep-ph/0210205.

[47] All details can be found at: L3 SUSY Working Group, http://13.web.cern.ch/13/analysis/susy/

M. Acciari, et al., L3 Collaboration, Phys. Lett. B472 (2000) 420;

L3 Collaboration, L3 Note2707 (2003).

[48] The LEP SUSY Working Group, http://lepsusy.web.cern.ch/lepsusy/.

[49] The limit on the LSP in MSUGRA can be found at http://lepsusy.web.cern.ch/lepsusy/www/lspmsugrasummer02/02-06.21/mSUGRA 208.html.

[50] G. Bélanger, F. Boudjema, F. Donato, R. Godbole, and S. Rosier-Lees, Nucl. Phys. B581 (2000) 3-33, hep-ph/0002039.

[51] S. Heinemeyer, W. Hollik, and G. Weiglein, Comput. Phys. Commun. 124 (2000) 76-89, hep-ph/9812320.

[52] A. Dedes and P. Slavich, Nucl. Phys. B657 (2003) 333-354, hep-ph/0212132.

[53] A. Heister, et al., ALEPH Collaboration, Phys. Lett. B526 (2002) 191; hep-ex/0201014. 
[54] ALEPH Collaboration, DELPHI Collaboration, L3 Collaboration, OPAL Collaboration, LHWG Note/2001-04; hep-ex/0107030.

[55] J. Guasch, P. Hafliger, and M. Spira, Phys. Rev. D68 (2003) 115001, hep-ph/0305101.

[56] F. Jegerlehner, Nucl. Phys. Proc. Suppl. 126 (2004) 325-334, hep-ph/0310234.

[57] K. Hagiwara, A. D. Martin, D. Nomura, and T. Teubner, Phys. Lett. B557 (2003) 69-75, hep-ph/0209187.

[58] J. F. de Troconiz and F. J. Yndurain, hep-ph/0402285.

[59] A. Nyffeler, Nucl. Phys. Proc. Suppl. 121 (2003) 187-190, hep-ph/0209329.

[60] M. Knecht, S. Peris, M. Perrottet, and E. De Rafael, JHEP 11 (2002) 003, hep-ph/0205102.

[61] M. Knecht, A. Nyffeler, M. Perrottet, and E. De Rafael, Phys. Rev. Lett. 88 (2002) 071802, hep-ph/0111059.

[62] M. Knecht and A. Nyffeler, Phys. Rev. D65 (2002) 073034, hep-ph/0111058.

[63] A. L. Kagan and M. Neubert, Eur. Phys. J. C7 (1999) 5-27, hep-ph/9805303.

[64] K. Chetyrkin, M. Misiak and M. Munz,Phys. Lett. B400 (1997) 206, Erratum-ibid. B425 (1997) 414; hep-ph/9612313.

[65] M. Ciuchini, G. Degrassi, P. Gambino, and G. F. Giudice, Nucl. Phys. B527 (1998) 21-43, hep-ph/9710335.

[66] G. Degrassi, P. Gambino and G. F. Giudice, JHEP 0012 (2000) 009, hep-ph/0009337.

[67] P. Gambino and M. Misiak, Nucl. Phys. B611 (2001) 338, hep-ph/0104034.

[68] B. Aubert et. al., The BABAR Collaboration, hep-ex/0207074 (2002);

B. Aubert et. al., The BABAR Collaboration, hep-ex/0207076 (2002).

[69] D. Cronin-Hennessy et. al., CLEO Collaboration Phys. Rev. Lett. 87 (2001) 251808, hep-ex/0108033.

[70] K. Abe et al., (BELLE Collaboration), Phys. Lett. B511 (2001) 151; H. Tajima, (BELLE Collaboration) Talk given at the 20th Lepton-Photon Conference, hep-ex/0111037.

[71] R. Barate et al, ALEPH Collaboration, Phys. Lett. 429 (1998) 169.

[72] C. Jessop, SLAC-PUB-9610.

[73] K. Anikeev et al., FERMILAB-Pub-01/197; hep-ph/0201071. Present limit is from F. Aben et al., CDF Collaboration, Phys. Rev. D57 (1998) 3811.

[74] C. Bobeth, T. Ewerth, F. Kruger and J. Urban, Phys. Rev. D64 (2001) 074014; hep-ph/0104284. 
[75] A. Dedes, H.K. Dreiner and U.Nierste, Phys. Rev. Lett. 87 (2001) 251804, hep-ph/0108037.

[76] A. Czarnecki and W. J. Marciano, Phys. Rev. D64 (2001) 013014, hep-ph/0102122. L. L. Everett, G. L. Kane, S. Rigolin and L. Wang, Phys. Rev. Lett. 86 (2001) 3484, hep-ph/0102145.

J. L. Feng and K. T. Matchev, Phys. Rev. Lett. 86 (2001) 3480, hep-ph/0102146.

E. A. Baltz and P. Gondolo, Phys. Rev. Lett. 86 (2001) 5004, hep-ph/0102147,

U. Chattopadhyay and P. Nath, Phys.Rev.Lett. 86 (2001) 5854, hep-ph/0102157.

J. Ellis, D. V. Nanopoulos and K. A. Olive, Phys. Lett. B508 (2001) 65;

hep-ph/0102331.

R. Arnowitt, B. Dutta, B. Hu and Y. Santoso, Phys. Lett. B505 (2001) 177, hep-ph/0102344.

S. P. Martin and J. D. Wells, Phys. Rev. D64 (2001) 035003, hep-ph/0103067.

H. Baer, C. Balazs, J. Ferrandis and X. Tata, Phys. Rev. D64 (2001) 035004, hep-ph/0103280.

D.G. Cerdeño, E. Gabrielli, S. Khalil, C. Muñoz and

E. Torrente-Lujan hep-ph/0104242

[77] K. Hagiwara et. al., Particle Data Group Collaboration, Phys. Rev. D66 (2002) 010001.

[78] G. Bélanger, F. Boudjema, A. Pukhov, A. Semenov, Talk at 4th International Workshop on the Identification of Dark Matter (IDM 2002), York, England, 2-6 Sep 2002, hep-ph/0210327

[79] R. Arnowitt, B. Dutta, T. Kamon, and M. Tanaka, Phys. Lett. B538 (2002) 121-129, hep-ph/0203069.

[80] The CDF Collaboration, the D0 Collaboration and the Tevatron Electroweak Working Group; hep-ex/0404010.

[81] P. Bambade, M. Berggren, F. Richard, and Z. Zhang, hep-ph/0406010.

[82] H.-U. Martyn, hep-ph/0406123.

[83] M. E. Gomez, T. Ibrahim, P. Nath, and S. Skadhauge, hep-ph/0404025.

[84] H. Baer, A. Belyaev, T. Krupovnickas, and X. Tata, JHEP 02 (2004) 007, hep-ph/0311351.

[85] H. Baer, A. Belyaev, T. Krupovnickas, and J. O'Farrill, hep-ph/0405210.

[86] C. Boehm, A. Djouadi, M. Drees, Phys. Rev.D62 (2000) 035012, hep-ph/9911496.

[87] Y. Santoso, Nucl. Phys. Proc. Suppl. 124 (2003) 166-169, hep-ph/0205026.

[88] H. Baer, C. Balazs, A. Belyaev, T. Krupovnickas, and X. Tata, JHEP 06 (2003) 054, hep-ph/0304303.

[89] H. Baer et. al., JHEP 05 (2002) 061, hep-ph/0204108.

[90] R. Arnowitt, B. Dutta, hep-ph/0204187

R. Arnowitt, B. Dutta, Y. Santoso, hep-ph/0010244. 
[91] D. G. Cerdeno and C. Munoz, hep-ph/0405057.

[92] A. Birkedal-Hansen, hep-ph/0306144.

[93] V. Bertin, E. Nezri, and J. Orloff, JHEP 02 (2003) 046, hep-ph/0210034.

[94] M. K. Gaillard, B. D. Nelson, and Y.-Y. Wu, Phys. Lett. B459 (1999) 549-556, hep-th/9905122.

[95] J. A. Bagger, T. Moroi, and E. Poppitz, JHEP 04 (2000) 009, hep-th/9911029.

[96] A. Birkedal-Hansen and B. D. Nelson, Phys. Rev. D67 (2003) 095006, hep-ph/0211071.

[97] H. Baer, C. Balazs, A. Belyaev, and J. O'Farrill, JCAP 0309 (2003) 007, hep-ph/0305191.

[98] P. Salati, Phys. Lett. B571 (2003) 121-131, astro-ph/0207396.

[99] D. B. Cline, H.-g. Wang, and Y. Seo, eConf C010630 (2001) E108, astro-ph/0108147. 